\title{
A characteristic map for compact quantum groups
}

\author{
Atabey Kaygun $^{1} \cdot$ Serkan Sütlï $^{2}$
}

Received: 24 December 2015 / Accepted: 3 June 2016 / Published online: 23 June 2016

(C) Tbilisi Centre for Mathematical Sciences 2016

\begin{abstract}
We show that if $G$ is a compact Lie group and $\mathfrak{g}$ is its Lie algebra, then there is a map from the Hopf-cyclic cohomology of the quantum enveloping algebra $U_{q}(\mathfrak{g})$ to the twisted cyclic cohomology of quantum group algebra $\mathcal{O}\left(G_{q}\right)$. We also show that the Schmüdgen-Wagner index cocycle associated with the volume form of the differential calculus on the standard Podleś sphere $\mathcal{O}\left(S_{q}^{2}\right)$ is in the image of this map.
\end{abstract}

Keywords Compact quantum group algebra . Characteristic map .

Cyclic cohomology

\section{Introduction}

Given a compact Lie group $G$ and its Lie algebra $\mathfrak{g}$, there is a characteristic map of the form $H P^{*}\left(U(\mathfrak{g}), k_{\delta}\right) \rightarrow H P^{*}(\mathcal{O}(G))$ coming from the Connes-Moscovici theory [6]. Here, the domain of the map depends on the Lie algebra homology of $\mathfrak{g}$, and the range is the ordinary periodic algebra cyclic cohomology of the algebra of regular functions on $G$, which depends on the de Rham homology of $G$. We refer the reader to Sect. 2.10 for details. In this paper we develop a $q$-analogue of this map. To

Communicated by Jonathan Rosenberg.

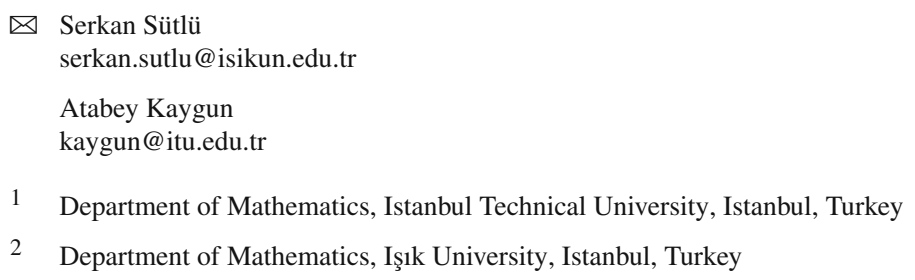


be precise, in Theorem 3.2 we show that for a compact quantum group algebra $\mathcal{O}\left(G_{q}\right)$ and its quantum enveloping algebra $U_{q}(\mathfrak{g})$ there is a morphism in cohomology of the form

$$
H C^{*}\left(U_{q}(\mathfrak{g}),{ }^{\sigma} k\right) \rightarrow H C_{\sigma^{-1}}^{*}\left(\mathcal{O}\left(G_{q}\right)\right)
$$

whose domain is the Hopf-cyclic cohomology of $U_{q}(\mathfrak{g})$ with coefficients in the modular pair in involution (MPI) determined by [25, Proposition 6.1.6], and whose range is the twisted cyclic cohomology of $\mathcal{O}\left(G_{q}\right)$ viewed as an algebra. Recall that the ConnesMoscovici characteristic map can be viewed as a cup product [18, 19,24,32]. Our key observation is that when we write the analogous cup product using the Haar functional of a compact quantum group, the modularity property of the Haar functional [25, Proposition 11.34] gives us the twisted algebra cyclic cohomology in the range in (1.1) in contrast to the Connes-Moscovici case where the range is the ordinary algebra cyclic cohomology. We further observe that one can untwist the cohomology with an appropriate additional cup product, but this procedure brings in a degree shift. The shift coming from the untwisting cup product explains the dimension drop phenomenon observed in [12], and the degree shift phenomenon observed in [13]. We refer the reader to Sect. 2.9 and Corollary 3.4.

We show the non-triviality of the characteristic homomorphism (1.1) in Sect. 4. We first recall that in [30] Masuda, Nakagami and Watanabe calculated the classical Hochschild and cyclic cohomology of $\mathcal{O}\left(S L_{q}(2)\right)$ using an explicit resolution. Then, in Proposition 4.6 and Corollary 4.7 we recover one specific generator of the cyclic cohomology of $\mathcal{O}\left(S L_{q}(2)\right)$ in the image of the characteristic homomorphism (1.1).

In analogy with the fact that Connes-Moscovici characteristic map allows the index computation of codimension- $n$ foliations to take place in the Hopf-cyclic cohomology of the Hopf algebra $\mathcal{H}_{n}$ of codimension- $n$ foliations, we introduce (1.1) to pull the index computation on the twisted cyclic cohomology of $\mathcal{O}\left(G_{q}\right)$ to the Hopf-cyclic cohomology $H C^{*}\left(U_{q}(\mathfrak{g}),{ }^{\sigma} k\right)$ of the Hopf algebra of $U_{q}(\mathfrak{g})$, which was computed in [22]. This fact, along with the quantum homogeneous space version of our characteristic map we develop in Sect. 5, turns the Hopf-cyclic cohomology of quantum groups into a useful tool detecting the index cocycles of such spaces. We use the equivariant characteristic map of [33] to show that in the case of the standard Podleś sphere, the characteristic homomorphism (1.1) descends to

$$
H C_{k\left[\sigma, \sigma^{-1}\right]}^{*}\left(U_{q}\left(s u_{2}\right),{ }^{\sigma^{-1}} k,{ }^{\sigma} k\right) \rightarrow H C_{\sigma^{-1}}^{*}\left(\mathcal{O}\left(S_{q}^{2}\right)\right)
$$

where the domain is now the equivariant Hopf-cyclic cohomology of the quantum enveloping algebra $U_{q}\left(s u_{2}\right)$. Moreover, we show that a $\sigma^{-1}$-twisted version of the Schmüdgen-Wagner quantum index cocycle of [34], see also [14], that computes the index of the Dirac operator on $\mathcal{O}\left(S U_{q}(2)\right)$ is in the image of (1.2). In the particular case of the (standard) Podleś sphere $\mathcal{O}\left(S_{q}^{2}\right) \subseteq \mathcal{O}\left(S U_{q}(2)\right)$, we further realize the Schmüdgen-Wagner index cocycle in the equivariant Hopf-cyclic cohomology [33] of $U_{q}\left(s u_{2}\right)$. 


\subsection{Notation and conventions}

We use a base field $k$ of characteristic 0 . WLOG one can assume $k=\mathbb{R}$. We are going to use $\mathbf{C B}$ and $\mathbf{C H}$ to denote respectively the bar and the Hochschild complexes associated with a (co)cyclic module. In the same vein, we use $H H, H C$ and $H P$ to denote respectively the Hochschild, the cyclic and the periodic cyclic (co)homology of a (co)cyclic module. We are going to use $\operatorname{Cotor}_{C}^{*}$ to denote the right derived functor of the (left exact) monoidal product $\square_{C}$ in the category of $C$-comodules of a coassociative counital coalgebra $C$. A coextension $\pi: C \rightarrow D$ is an epimorphism of (counital) coalgebras.

\section{Preliminaries}

In this section we recall the basic material that will be needed in the sequel.

\subsection{Cobar and Hochschild complexes}

In this subsection we recall the definition of the cobar complex of a coalgebra $\mathcal{C}$, as well as the Cotor-groups associated to a coalgebra $\mathcal{C}$ and a pair $(V, W)$ of $\mathcal{C}$-comodules of opposite parity.

Let $\mathcal{C}$ be a coassociative coalgebra. Following [2,9,21], the cobar complex of $\mathcal{C}$ is defined to be the differential graded space

$$
\mathbf{C B}^{*}(\mathcal{C}):=\bigoplus_{n \geqslant 0} \mathcal{C}^{\otimes n+2}
$$

with the differentials $d: \mathbf{C B}^{n}(\mathcal{C}) \longrightarrow \mathbf{C B}^{n+1}(\mathcal{C})$

$$
d\left(c^{0} \otimes \cdots \otimes c^{n+1}\right)=\sum_{j=0}^{n}(-1)^{j} c^{0} \otimes \cdots \otimes \Delta\left(c^{j}\right) \otimes \cdots \otimes c^{n+1} .
$$

Let $\mathcal{C}^{e}:=\mathcal{C} \otimes \mathcal{C}^{\text {cop }}$ be the enveloping coalgebra of $\mathcal{C}$. In case $\mathcal{C}$ is counital, the cobar complex $\mathbf{C B}^{*}(\mathcal{C})$ yields a $\mathcal{C}^{e}$-injective resolution of the (left) $\mathcal{C}^{e}$-comodule $\mathcal{C}$, see for instance [9].

Following the terminology of [20], for a pair $(V, W)$ of two $\mathcal{C}$-comodules of opposite parity (say, $V$ is a right $\mathcal{C}$-comodule and $W$ is a left $\mathcal{C}$-comodule,) we call the complex

$$
\left(\mathbf{C B}^{*}(V, \mathcal{C}, W), d\right), \quad \mathbf{C B}^{*}(V, \mathcal{C}, W):=V \square_{\mathcal{C}} \mathbf{C B}^{*}(\mathcal{C}) \square_{\mathcal{C}} W
$$

where we define $d: \mathbf{C B}^{n}(V, \mathcal{C}, W) \rightarrow \mathbf{C B}^{n+1}(V, \mathcal{C}, W)$ 


$$
\begin{aligned}
d\left(v \otimes c^{1} \otimes \cdots \otimes c^{n} \otimes w\right)= & v_{<0>} \otimes v_{<1>} \otimes c^{1} \otimes \cdots \otimes c^{n} \otimes w \\
& +\sum_{j=1}^{n}(-1)^{j} c^{1} \otimes \cdots \otimes \Delta\left(c^{j}\right) \otimes \cdots \otimes c^{n} \otimes w \\
& +(-1)^{n+1} v \otimes c^{1} \otimes \cdots \otimes c^{n} \otimes w_{<-1>} \otimes w_{<0>}
\end{aligned}
$$

the two-sided (cohomological) cobar complex of the coalgebra $\mathcal{C}$. In case $\mathcal{C}$ is a counital coalgebra, the Cotor-groups of a pair $(V, W)$ of $\mathcal{C}$-comodules of opposite parity can be computed from

$$
\operatorname{Cotor}_{\mathcal{C}}^{*}(V, W)=H^{*}\left(\mathbf{C B}^{*}(V, \mathcal{C}, W), d\right)
$$

We next recall the Hochschild cohomology of a coalgebra $\mathcal{C}$ with coefficients in the $\mathcal{C}$-bicomodule (equivalently $\mathcal{C}^{e}$-comodule) $V$, from [9], as the cohomology of the complex

$$
\mathbf{C H}^{*}(\mathcal{C}, V)=\bigoplus_{n \geqslant 0} \mathbf{C H}^{n}(\mathcal{C}, V), \quad \mathbf{C H}^{n}(\mathcal{C}, V):=V \otimes \mathcal{C}^{\otimes n}
$$

with the differential $b: \mathbf{C H}^{n}(\mathcal{C}, V) \rightarrow \mathbf{C H}^{n+1}(\mathcal{C}, V)$ defined as

$$
\begin{aligned}
b\left(v \otimes c^{1} \otimes \cdots \otimes c^{n}\right)= & v_{<0>} \otimes v_{<1>} \otimes c^{1} \otimes \cdots \otimes c^{n} \\
& +\sum_{k=1}^{n}(-1)^{k} c^{1} \otimes \cdots \otimes \Delta\left(c^{k}\right) \otimes \cdots \otimes c^{n} \\
& +(-1)^{n+1} v_{<0>} \otimes c^{1} \otimes \cdots \otimes c^{n} \otimes v_{<-1>} .
\end{aligned}
$$

We identify $\mathbf{C B}^{n}(\mathcal{C})$ with $\mathcal{C}^{e} \otimes \mathcal{C}^{\otimes n}$ as left $\mathcal{C}^{e}$-comodules for $n>0$ via

$$
c^{0} \otimes \cdots \otimes c^{n+1} \mapsto\left(c^{0} \otimes c^{n+1}\right) \otimes c^{1} \otimes \cdots \otimes c^{n} .
$$

The left $\mathcal{C}^{e}$-comodule structure on $\mathbf{C B}^{n}(\mathcal{C})=\mathcal{C}^{\otimes n+2}$ is given by

$$
\nabla\left(c^{0} \otimes \cdots \otimes c^{n+1}\right)=\left(c_{(1)}^{0} \otimes c_{(2)}^{n+1}\right) \otimes\left(c_{(2)}^{0} \otimes c^{1} \otimes \cdots \otimes c^{n} \otimes c_{(1)}^{n+1}\right),
$$

and on $\mathcal{C}^{e} \otimes \mathcal{C}^{\otimes n}$ by

$$
\nabla\left(\left(c \otimes c^{\prime}\right) \otimes\left(c^{1} \otimes \cdots \otimes c^{n}\right)\right)=\left(c_{(1)} \otimes c_{(2)}^{\prime}\right) \otimes\left(c_{(2)} \otimes c_{(1)}^{\prime}\right) \otimes\left(c^{1} \otimes \cdots \otimes c^{n}\right) .
$$

This yields an isomorphism of the form

$$
\left(\mathbf{C H}^{*}(\mathcal{C}, V), b\right) \cong\left(V \square_{\mathcal{C}^{e}} \mathbf{C B}^{*}(\mathcal{C}), d\right)
$$

on the chain level. In case $\mathcal{C}$ is counital one can interpret the Hochschild cohomology of $\mathcal{C}$ with coefficients in $V$ in terms of Cotor-groups as

$$
H^{*}(\mathcal{C}, V)=H^{*}\left(\mathbf{C H}^{*}(\mathcal{C}, V), b\right)=\operatorname{Cotor}_{\mathcal{C}^{e}}^{*}(V, \mathcal{C})
$$


or more generally,

$$
H^{*}(\mathcal{C}, V)=H^{*}\left(V \square_{\mathcal{C}^{e}} Y^{*}\right)
$$

for any coflat resolution $Y^{*}$ of $\mathcal{C}$ via left $\mathcal{C}^{e}$-comodules.

\subsection{Cohomology of coextensions}

In this subsection we recall the main computational tool introduced in [22] which can be summarized as follows: Given a coflat coalgebra coextension $\mathcal{C} \rightarrow \mathcal{D}$, the coalgebra Hochschild cohomology of $\mathcal{C}$ can be computed relatively easily by means of the Hochschild cohomology of $\mathcal{D}$, and the relative cohomology of the coextension.

Let $\pi: \mathcal{C} \rightarrow \mathcal{D}$ be a coextension. We first introduce the auxiliary coalgebra $\mathcal{Z}:=\mathcal{C} \oplus \mathcal{D}$ with the comultiplication

$$
\Delta(y)=y_{(1)} \otimes y_{(2)} \quad \text { and } \quad \Delta(x)=x_{(1)} \otimes x_{(2)}+\pi\left(x_{(1)}\right) \otimes x_{(2)}+x_{(1)} \otimes \pi\left(x_{(2)}\right),
$$

and the counit

$$
\varepsilon(x+y)=\varepsilon(y),
$$

for any $x \in \mathcal{C}$ and $y \in \mathcal{D}$.

Next, let $V$ be a $\mathcal{C}$-bicomodule and let $\mathcal{C}$ be coflat both as a left and a right $\mathcal{D}$ comodule. Then via the short exact sequence

$$
0 \rightarrow \mathcal{D} \stackrel{i}{\rightarrow} \mathcal{Z} \stackrel{p}{\rightarrow} \mathcal{C} \rightarrow 0 \quad \text { where } i: y \mapsto(0, y) \quad p:(x, y) \mapsto x
$$

of coalgebras and [11, Lemma 4.10], we have

$$
H H^{n}(\mathcal{Z}, V) \cong H H^{n}(\mathcal{C}, V), \quad n \geqslant 0
$$

We next consider $\mathbf{C} \mathbf{H}^{*}(\mathcal{Z}, V)$ with the decreasing filtration

$$
F_{p}^{n+p}= \begin{cases}\bigoplus_{n_{0}+\cdots+n_{p}=n} V \otimes \mathcal{Z}^{\otimes n_{0}} \otimes \mathcal{C} \otimes \cdots \otimes \mathcal{Z}^{n_{p-1}} \otimes \mathcal{C} \otimes \mathcal{Z}^{\otimes n_{p}}, & p \geqslant 0 \\ 0, & p<0\end{cases}
$$

The associated spectral sequence is

$$
E_{0}^{i, j}=F_{i}^{i+j} / F_{i+1}^{i+j}=\bigoplus_{n_{0}+\cdots+n_{i}=j} V \otimes \mathcal{D}^{\otimes n_{0}} \otimes \mathcal{C} \otimes \cdots \otimes \mathcal{D}^{n_{i-1}} \otimes \mathcal{C} \otimes \mathcal{D}^{\otimes n_{i}},
$$

and by the coflatness assumption, on the vertical direction it computes

$$
H H^{j}\left(\mathcal{D}, \mathcal{C}^{\square_{\mathcal{D}} i} \square_{\mathcal{D}} V\right) .
$$

Hence we have the following. 
Theorem 2.1 Let $\pi: \mathcal{C} \rightarrow \mathcal{D}$ be a coalgebra coextension and $V=V^{\prime} \otimes V^{\prime \prime} a$ $\mathcal{C}$-bicomodule such that the left $\mathcal{C}$-comodule structure is given by $V^{\prime}$ and the right $\mathcal{C}$-comodule structure is given by $V^{\prime \prime}$. Let also $\mathcal{C}$ be coflat both as a left and a right $\mathcal{D}$-comodule. Then there is a spectral sequence whose $E_{1}$-term is of the form

$$
E_{1}^{i, j}=\operatorname{Cotor}_{\mathcal{D}}^{j}\left(V^{\prime \prime}, \mathcal{C}^{\square_{\mathcal{D}} i} \square_{\mathcal{D}} V^{\prime}\right)
$$

converging to $H H^{i+j}(\mathcal{C}, V)$.

A convenient set-up as a test case for our machinery is a principal coextension [1,35]. We assume $\mathcal{H}$ is a Hopf algebra with a bijective antipode, and $\mathcal{C}$ is a left $\mathcal{H}$ module coalgebra. Since $\mathcal{H}^{+}=\operatorname{ker} \varepsilon$ is the augmentation ideal of $\mathcal{H}$, if we define a quotient coalgebra by $\mathcal{D}:=\mathcal{C} / \mathcal{H}^{+} \mathcal{C}$ then by [35, Theorem II],

1. $\mathcal{C}$ is a projective left $\mathcal{H}$-module,

2. can $: \mathcal{H} \otimes \mathcal{C} \rightarrow \mathcal{C} \square_{\mathcal{D}} \mathcal{C}, h \otimes c \mapsto h \cdot c_{(1)} \otimes c_{(2)}$ is injective,

if and only if

3. $\mathcal{C}$ is faithfully flat left (and right) $\mathcal{D}$-comodule,

4. can $: \mathcal{H} \otimes \mathcal{C} \rightarrow \mathcal{C} \square_{\mathcal{D}} \mathcal{C}$ is an isomorphism.

\subsection{Cyclic cohomology of algebras}

We recall the cyclic cohomology of algebras [3-5,29]. Let $\mathcal{A}$ be an algebra and $M$ an $\mathcal{A}$-bimodule. Then the Hochschild cohomology $H H(\mathcal{A}, M)$ of $\mathcal{A}$ with coefficients in $M$ is the homology of the complex

$$
C(\mathcal{A}, M)=\bigoplus_{n \geqslant 0} C^{n}(\mathcal{A}, M),
$$

where $C^{n}(\mathcal{A}, M)$ is the space of all linear maps $\mathcal{A}^{\otimes n} \rightarrow M$ with the differential

$$
\begin{aligned}
b \varphi\left(a_{1}, \ldots, a_{n+1}\right)= & a_{1} \cdot \varphi\left(a_{2}, \ldots, a_{n+1}\right) \\
& +\sum_{k=1}^{n}(-1)^{k} \varphi\left(a_{1}, \ldots, a_{k} a_{k+1} \ldots, a_{n+1}\right) \\
& +(-1)^{n+1} \varphi\left(a_{1}, \ldots, a_{n}\right) \cdot a_{n+1} .
\end{aligned}
$$

The space $\mathcal{A}^{*}$ of all linear maps of the form $\mathcal{A} \rightarrow k$ is an $\mathcal{A}$-bimodule via $a \cdot \varphi \cdot b(c)=$ $\varphi(b c a)$ defined for every $\varphi \in \mathcal{A}^{*}$, and $a, b, c \in \mathcal{A}$. Hence, the complex $C\left(\mathcal{A}, \mathcal{A}^{*}\right)$ can be defined. If we identify $\varphi \in C^{n}\left(\mathcal{A}, \mathcal{A}^{*}\right)$ with

$$
\phi: \mathcal{A}^{\otimes n+1} \rightarrow k, \quad \phi\left(a_{0}, \alpha_{1}, \ldots, a_{n}\right):=\varphi\left(a_{1}, \ldots, a_{n}\right)\left(a_{0}\right),
$$

the coboundary map corresponds to 


$$
\begin{aligned}
b \phi\left(a_{0}, \ldots, a_{n+1}\right)= & \phi\left(a_{0} a_{1}, \ldots, a_{n+1}\right) \\
& +\sum_{k=1}^{n}(-1)^{k} \varphi\left(a_{0}, \ldots, a_{k} a_{k+1} \ldots, a_{n+1}\right) \\
& +(-1)^{n+1} \varphi\left(a_{n+1} a_{0}, \ldots, a_{n}\right) .
\end{aligned}
$$

With these definitions at hand, we define the cyclic cohomology $H_{\lambda}(\mathcal{A})$ of the algebra $\mathcal{A}$ as the homology of the subcomplex

$$
C_{\lambda}^{*}(\mathcal{A})=\bigoplus_{n \geqslant 0} C_{\lambda}^{n}\left(\mathcal{A}, \mathcal{A}^{*}\right)
$$

where

$$
C_{\lambda}^{n}\left(\mathcal{A}, \mathcal{A}^{*}\right):=\left\{\phi \in C^{n}\left(\mathcal{A}, \mathcal{A}^{*}\right) \mid \phi\left(a_{0}, \ldots, a_{n}\right)=(-1)^{n} \phi\left(a_{n}, a_{0}, \ldots, a_{n-1}\right)\right\}
$$

Equivalently, the cyclic cohomology $H C(\mathcal{A})$ of an algebra $\mathcal{A}$ can also be defined as the cyclic cohomology of the cocyclic module associated to

$$
C^{*}(\mathcal{A})=\bigoplus_{n \geqslant 0} C^{n}(\mathcal{A}), \quad C^{n}(\mathcal{A}):=\operatorname{Hom}\left(\mathcal{A}^{\otimes n+1}, k\right)
$$

by its own cofaces, codegeneracies and cyclic group actions. The coface maps $d_{k}$ : $C^{n}(\mathcal{A}) \rightarrow C^{n+1}(\mathcal{A})$ are defined for $0 \leqslant k \leqslant n+1$ as

$$
d_{k} \varphi\left(a^{0}, \ldots, a^{n+1}\right)= \begin{cases}\varphi\left(a^{0}, \ldots, a^{k} a^{k+1}, \ldots, a^{n+1}\right) & \text { if } 0 \leqslant k \leqslant n \\ \varphi\left(a^{n+1} a^{0}, a^{1}, \ldots, a^{n}\right) & \text { if } k=n+1\end{cases}
$$

The codegenerecy maps $s_{j}: C^{n}(\mathcal{A}) \rightarrow C^{n-1}(\mathcal{A})$ are defined for $0 \leqslant j \leqslant n-1$ as

$$
s_{j} \varphi\left(a^{0}, \ldots, a^{n-1}\right)=\varphi\left(a^{0}, \ldots, a^{j}, 1, a^{j+1}, \ldots, a^{n+1}\right),
$$

and finally the cyclic operators $t_{n}: C^{n}(\mathcal{A}) \rightarrow C^{n}(\mathcal{A})$ as

$$
t_{n} \varphi\left(a^{0}, \ldots, a^{n}\right)=\varphi\left(a^{n}, a^{0}, \ldots, a^{n-1}\right)
$$

The cyclic cohomology of $\mathcal{A}$ is defined to be the total cohomology of the associated first quadrant bicomplex $(C C(\mathcal{A}), b, B)$ where

$$
C C^{p, q}(\mathcal{A}):= \begin{cases}C^{q-p}(\mathcal{A}) & \text { if } q \geqslant p \geqslant 0 \\ 0 & \text { if } p>q\end{cases}
$$


with the algebra Hochschild coboundary operator $b: C C^{p, q}(\mathcal{A}) \rightarrow C C^{p, q+1}(\mathcal{A})$ which is given by

$$
b:=\sum_{i=0}^{q+1}(-1)^{i} d_{i}
$$

and the Connes boundary operator $B: C C^{p, q}(\mathcal{A}) \rightarrow C C^{p-1, q}(\mathcal{A})$ which is defined as

$$
B:=\left(\sum_{i=0}^{p}(-1)^{p i} t_{p}^{i}\right)\left(1+(-1)^{p} t_{p}\right) s_{p} .
$$

We recall that $H_{\lambda}^{*}(\mathcal{A}) \cong H C^{*}(\mathcal{A})$, since we assume throughout that the ground field $k$ is of characteristic 0 .

\subsection{Twisted cyclic cohomology}

We next briefly recall from [26] the twisted cyclic cohomology of an algebra $\mathcal{A}$ by an automorphism $\sigma: \mathcal{A} \rightarrow \mathcal{A}$. Let $C^{n}(\mathcal{A})$ be the set of all linear maps $\mathcal{A}^{\otimes n+1} \rightarrow k$. Then, the complex

$$
C_{\sigma}^{*}(\mathcal{A})=\bigoplus_{n \geqslant 0} C_{\sigma}^{n}(\mathcal{A})
$$

where

$$
C_{\sigma}^{n}(\mathcal{A})=\left\{\phi \in C^{n}(\mathcal{A}) \mid \phi\left(a_{0}, \ldots, a_{n}\right)=(-1)^{n} \phi\left(\sigma\left(a_{n}\right), a_{0}, \ldots, a_{n-1}\right)\right\},
$$

is closed under the twisted Hochschild differential,

$$
\begin{aligned}
b \phi\left(a_{0}, \ldots, a_{n+1}\right)= & \phi\left(a_{0} a_{1}, \ldots, a_{n+1}\right) \\
& +\sum_{k=1}^{n}(-1)^{k} \varphi\left(a_{0}, \ldots, a_{k} a_{k+1} \ldots, a_{n+1}\right) \\
& +(-1)^{n+1} \varphi\left(\sigma\left(a_{n+1}\right) a_{0}, \ldots, a_{n}\right) .
\end{aligned}
$$

Then the homology of the complex (2.12) with the differential map (2.14) is called the $\sigma$-twisted cyclic cohomology of the algebra $\mathcal{A}$.

Equivalently, the $\sigma$-twisted cyclic cohomology $H C_{\sigma}^{*}(\mathcal{A})$ of the algebra $\mathcal{A}$ is computed by the cocyclic object

$$
C_{\sigma}^{n}(\mathcal{A})=\left\{\phi \in C^{n}(\mathcal{A}) \mid \phi\left(\sigma\left(a_{0}\right), \ldots, \sigma\left(a_{n}\right)\right)=\phi\left(a_{0}, \ldots, a_{n}\right)\right\}
$$

given by the coface maps $d_{k}: C_{\sigma}^{n}(\mathcal{A}) \rightarrow C_{\sigma}^{n+1}(\mathcal{A})$ for $0 \leqslant k \leqslant n+1$,

$$
d_{k} \varphi\left(a^{0}, \ldots, a^{n+1}\right)= \begin{cases}\varphi\left(a^{0}, \ldots, a^{k} a^{k+1}, \ldots, a^{n+1}\right) & \text { if } 0 \leqslant k \leqslant n \\ \varphi\left(\sigma\left(a^{n+1}\right) a^{0}, a^{1}, \ldots, a^{n}\right) & \text { if } k=n+1\end{cases}
$$


the codegeneracy maps $s_{j}: C_{\sigma}^{n}(\mathcal{A}) \rightarrow C_{\sigma}^{n-1}(\mathcal{A})$ for $0 \leqslant j \leqslant n-1$ as

$$
s_{j} \varphi\left(a^{0}, \ldots, a^{n-1}\right)=\varphi\left(a^{0}, \ldots, a^{j}, 1, a^{j+1}, \ldots, a^{n+1}\right),
$$

and finally the cyclic operators $t_{n}: C_{\sigma}^{n}(\mathcal{A}) \rightarrow C_{\sigma}^{n}(\mathcal{A})$

$$
t_{n} \varphi\left(a^{0}, \ldots, a^{n}\right)=\varphi\left(\sigma\left(a^{n}\right), a^{0}, \ldots, a^{n-1}\right) .
$$

\subsection{Hopf-cyclic cohomology}

In this subsection we recall the Hopf-cyclic cohomology for Hopf algebras from [7, Sects. 3, 4], see also [8, Sect. 2].

Let $\mathcal{H}$ be a Hopf algebra with a modular pair $(\delta, \sigma)$ in involution (MPI). In other words, $\delta: \mathcal{H} \rightarrow k$ is a character, and $\sigma \in \mathcal{H}$ a group-like satisfying the modularity condition

$$
\delta(\sigma)=1 \quad \text { and } \quad S_{\delta}^{2}=\operatorname{Ad}_{\sigma}, \quad \text { where } S_{\delta}(h):=\delta\left(h_{(1)}\right) S\left(h_{(2)}\right),
$$

for all $h \in H$. Then the Hopf-cyclic cohomology $H C^{*}(\mathcal{H}, \delta, \sigma)$ of $\mathcal{H}$, relative to the pair $(\delta, \sigma)$ is defined to be the cyclic cohomology of the cocyclic module [6]

$$
C^{*}(\mathcal{H}, \delta, \sigma)=\bigoplus_{n \geqslant 0} C^{n}(\mathcal{H}, \delta, \sigma), \quad C^{n}(\mathcal{H}, \delta, \sigma):=\mathcal{H}^{\otimes n}
$$

The coface operators $d_{i}: C^{n}(\mathcal{H}, \delta, \sigma) \rightarrow C^{n+1}(\mathcal{H}, \delta, \sigma)$ are defined for $0 \leq i \leq n+1$ as

$$
d_{i}\left(h^{1} \otimes \cdots \otimes h^{n}\right)= \begin{cases}1 \otimes h^{1} \otimes \cdots \otimes h^{n}, & \text { if } i=0, \\ h^{1} \otimes \cdots \otimes h_{(1)}^{i} \otimes h_{(2)}^{i} \otimes \cdots \otimes h^{n}, & \text { if } 0 \leqslant i \leqslant n \\ h^{1} \otimes \cdots \otimes h^{n} \otimes \sigma, & \text { if } i=n+1\end{cases}
$$

We define the codegeneracy operators $s_{j}: C^{n}(\mathcal{H}, \delta, \sigma) \rightarrow C^{n-1}(\mathcal{H}, \delta, \sigma)$ for $0 \leq$ $j \leq n-1$ as

$$
s_{j}\left(h^{1} \otimes \cdots \otimes h^{n}\right)=h^{1} \otimes \cdots \otimes \varepsilon\left(h^{j+1}\right) \otimes \cdots \otimes h^{n},
$$

and the cyclic operators $t_{n}: C^{n}(\mathcal{H}, \delta, \sigma) \rightarrow C^{n}(\mathcal{H}, \delta, \sigma)$ as

$$
t_{n}\left(h^{1} \otimes \cdots \otimes h^{n}\right)=S_{\delta}\left(h^{1}\right) \cdot\left(h^{2} \otimes \cdots \otimes h^{n} \otimes \sigma\right)
$$




\subsection{Connes-Moscovici characteristic map}

Let us next recall the Connes-Moscovici characteristic homomorphism, [6,7]. Let $\mathcal{A}$ be a $\mathcal{H}$-module algebra, that is, for all $h \in \mathcal{H}$ and for all $a, b \in \mathcal{A}$,

$$
h(a b)=h_{(1)}(a) h_{(2)}(b), \quad h(1)=\varepsilon(h) 1 .
$$

Then a linear form $\tau: \mathcal{A} \rightarrow k$ is called a $\sigma$-trace if

$$
\tau(a b)=\tau(b \sigma(a))
$$

and the $\sigma$-trace $\tau: \mathcal{A} \rightarrow k$ is called $\delta$-invariant if

$$
\tau(h(a))=\delta(h) \tau(a),
$$

for all $h \in \mathcal{H}$ and $a, b \in \mathcal{A}$.

Now let $\mathcal{H}$ be a Hopf algebra with a $\operatorname{MPI}(\delta, \sigma)$, and $\mathcal{A}$ an $\mathcal{H}$-module algebra equipped with a $\delta$-invariant $\sigma$-trace. It follows then that the morphisms $\chi_{\tau}: C^{n}(\mathcal{H}, \delta, \sigma) \rightarrow C^{n}(\mathcal{A})$,

$$
\chi_{\tau}\left(h^{1} \otimes \cdots \otimes h^{n}\right)\left(a^{0}, \ldots, a^{n}\right):=\tau\left(a^{0} h^{1}\left(a^{1}\right) \ldots h^{n}\left(a^{n}\right)\right)
$$

induce a characteristic homomorphism on the cohomology $\chi_{\tau}: H C(\mathcal{H}, \delta, \sigma) \rightarrow$ $H C(\mathcal{A})$.

\subsection{Hopf-cyclic cohomology of module algebras}

We now recall from [15] the Hopf-cyclic cohomology theory for the module algebra symmetry. In order to define the coefficient spaces, we first note that a modular pair in involution is an example of a one dimensional stable anti-Yetter Drinfeld (SAYD) module [16]. In general, a right module-left comodule $V$ over a Hopf algebra $\mathcal{H}$ is called a right-left SAYD module over $\mathcal{H}$ if

$$
\nabla(h \cdot v)=S\left(h_{(3)}\right) v_{<-1>} h_{(1)} \otimes v_{<0>}, \quad v_{<0>} \cdot v_{<-1>}=v
$$

for any $v \in V$ and any $h \in \mathcal{H}$. Here $\nabla: V \rightarrow \mathcal{H} \otimes V$ given by $v \mapsto v_{<-1>} \otimes v_{<0>}$ refers to the left $\mathcal{H}$-coaction on $V$.

Let $\mathcal{A}$ be an $\mathcal{H}$-module algebra and $V$ a SAYD module over $\mathcal{H}$. We recall from $[15,32]$ that the graded space

$$
C_{\mathcal{H}}^{*}(\mathcal{A}, V)=\bigoplus_{n \geqslant} C_{\mathcal{H}}^{n}(\mathcal{A}, V), \quad C_{\mathcal{H}}^{n}(\mathcal{A}, V):=\operatorname{Hom}_{\mathcal{H}}\left(V \otimes \mathcal{A}^{\otimes n+1}, k\right)
$$


becomes a cocyclic module via the coface maps $\partial_{i}: C_{\mathcal{H}}^{n}(\mathcal{A}, V) \rightarrow C_{\mathcal{H}}^{n+1}(\mathcal{A}, V)$, defined for $0 \leqslant i \leqslant n+1$

$$
\partial_{i} \varphi\left(v, a^{0}, \ldots, a^{n+1}\right)= \begin{cases}\varphi\left(v, a^{0}, \ldots, a^{i} a^{i+1}, \ldots, a^{n+1}\right), & \text { if } 0 \leqslant i \leqslant n \\ \varphi\left(v_{<0>}, S^{-1}\left(v_{<-1>}\right)\left(a^{n+1}\right) a^{0}, a^{1}, \ldots, a^{n}\right), & \text { if } i=n+1,\end{cases}
$$

the codegenerecy maps $s_{j}: C_{\mathcal{H}}^{n}(\mathcal{A}, V) \rightarrow C_{\mathcal{H}}^{n-1}(\mathcal{A}, V)$, defined for $0 \leqslant j \leqslant n-1$ by

$$
s_{j} \varphi\left(v, a^{0}, \ldots, a^{n-1}\right)=\varphi\left(v, a^{0}, \ldots, a^{j}, 1, a^{j+1}, \ldots, a^{n-1}\right),
$$

and the cyclic operators $t_{n}: C_{\mathcal{H}}^{n}(\mathcal{A}, V) \rightarrow C_{\mathcal{H}}^{n}(\mathcal{A}, V)$,

$$
t_{n} \varphi\left(v, a^{0}, \ldots, a^{n}\right)=\varphi\left(v_{<0>}, S^{-1}\left(v_{<-1>}\right)\left(a^{n}\right), a^{0}, \ldots, a^{n-1}\right) .
$$

The cyclic homology of this cocyclic module is called the Hopf-cyclic cohomology of the $\mathcal{H}$-module algebra $\mathcal{A}$ with coefficients, and is denoted by $H C_{\mathcal{H}}^{*}(\mathcal{A}, V)$. We note from [15] that if $\sigma \in \operatorname{Aut}(\mathcal{A})$, then with the Hopf algebra $\mathcal{H}=k\left[\sigma, \sigma^{-1}\right]$ of Laurent polynomials and $V={ }^{-1} k$ we recover the twisted cyclic cohomology.

\subsection{Hopf-cyclic cohomology of module coalgebras}

Let us next recall the Hopf-cyclic cohomology of module coalgebras with SAYD coefficients. Let $\mathcal{C}$ be a left $\mathcal{H}$-module coalgebra. That is, $\mathcal{H}$ acts on $\mathcal{C}$ such that

$$
\Delta(h \cdot c)=h_{(1)} \cdot c_{(1)} \otimes h_{(2)} \cdot c_{(2)}, \quad \varepsilon(h \cdot c)=\varepsilon(h) \varepsilon(c),
$$

for any $h \in \mathcal{H}$, and any $c \in \mathcal{C}$. Let also $V$ be a right-left SAYD module over $\mathcal{H}$. Then the Hopf-cyclic cohomology of $\mathcal{C}$ under the symmetry of $\mathcal{H}$ is given by the cocyclic module of the coface operators $\partial_{i}: C_{\mathcal{H}}^{n}(\mathcal{C}, V) \rightarrow C_{\mathcal{H}}^{n+1}(\mathcal{C}, V)$ defined for $0 \leq i \leq n+1$ by

$$
\begin{aligned}
\partial_{i} & \left(v \otimes_{\mathcal{H}} c^{0} \otimes \cdots \otimes c^{n}\right) \\
& = \begin{cases}v \otimes_{\mathcal{H}} c^{0} \otimes \cdots \otimes c_{(1)}^{i} \otimes c_{(2)}^{i} \otimes \cdots \otimes c^{n}, & \text { if } 0 \leqslant i \leqslant n, \\
v_{<0>} \otimes_{\mathcal{H}} c_{(2)}^{0} \otimes c^{1} \otimes \cdots \otimes c^{n} \otimes v_{<-1>} \cdot c_{(1)}^{0}, & \text { if } i=n+1,\end{cases}
\end{aligned}
$$

the codegeneracy operators $\sigma_{j}: C_{\mathcal{H}}^{n}(\mathcal{C}, V) \rightarrow C_{\mathcal{H}}^{n-1}(\mathcal{C}, V)$ for $0 \leq j \leq n-1$

$$
\sigma_{j}\left(v \otimes \mathcal{H} c^{0} \otimes \cdots \otimes c^{n}\right)=v \otimes_{\mathcal{H}} c^{0} \otimes \cdots \otimes \varepsilon\left(c^{j+1}\right) \otimes \cdots \otimes c^{n},
$$

and the cocyclic operators $\tau_{n}: C_{\mathcal{H}}^{n}(\mathcal{C}, V) \rightarrow C_{\mathcal{H}}^{n}(\mathcal{C}, V)$

$$
\tau_{n}\left(v \otimes_{\mathcal{H}} c^{0} \otimes \cdots \otimes c^{n}\right)=v_{<0>} \otimes_{\mathcal{H}} c^{1} \otimes \cdots \otimes v_{<-1>} \cdot c^{0} .
$$


The cyclic homology of this cocyclic module is denoted by $H C_{\mathcal{H}}^{*}(\mathcal{C}, V)$. In particular, if $\mathcal{C}=\mathcal{H}$ which is considered as a left $\mathcal{H}$-module coalgebra by the left regular action of $\mathcal{H}$ on itself, the Hopf-cyclic cohomology with coefficients of the Hopf algebra $\mathcal{H}$ is denoted by $H C^{*}(\mathcal{H}, V)$. If, furthermore, $V={ }^{\sigma} k_{\delta}$ the one dimensional SAYD module by a $\operatorname{MPI}(\delta, \sigma)$ of $\mathcal{H}$, the cocyclic structure given by (2.25), (2.26) and (2.27) reduces to the one given by $(2.17),(2.18)$ and $(2.19),[15]$.

\subsection{The characteristic map and untwisting}

Let us recall from [18, Theorem 2.8] and [32, Proposition 2.3] that if $\mathcal{A}$ is a left $\mathcal{H}$ module algebra and $V$ a right-left SAYD module over $\mathcal{H}$, then there is a cup product

$$
\cup: H C_{\mathcal{H}}^{p}(\mathcal{A}, V) \otimes H C^{q}(\mathcal{H}, V) \rightarrow H C^{p+q}(\mathcal{A})
$$

On the level of Hochschild cohomology, it is given by the formula

$$
\left(\varphi \cup\left(v \otimes h^{1} \otimes \cdots \otimes h^{p}\right)\right)\left(a^{0}, \ldots, a^{p+q}\right)=\varphi\left(v, a^{0} h^{1}\left(a^{1}\right) \ldots h^{p}\left(a^{p}\right), a^{p+1}, \ldots, a^{p+q}\right),
$$

and, following [24,32], in the level of cyclic cohomology by

$$
\begin{aligned}
(\varphi & \cup \widetilde{h})\left(a^{0}, \ldots, a^{p+q}\right) \\
\quad & =\sum_{\mu \in \operatorname{Sh}(q, p)}(-1)^{\mu} \partial_{\bar{\mu}(q)} \ldots \partial_{\bar{\mu}(1)} \varphi\left(\partial_{\bar{\mu}(q+p)} \ldots \partial_{\bar{\mu}(q+1)} \widetilde{h}\left(a^{0}, \ldots, a^{p+q}\right)\right),
\end{aligned}
$$

where $\widetilde{h}=v \otimes_{\mathcal{H}} h^{0} \otimes \cdots \otimes h^{p}, \bar{\mu}(\ell)=\mu(\ell)-1$, and $\operatorname{Sh}(q, p)$ denotes the set of all $(p, q)$-shuffles. We note also that, for a Hopf algebra $\mathcal{H}$ with a MPI $(\delta, \sigma)$, the cup product by a 0 -cocycle $\tau \in H C^{0}\left(\mathcal{H},{ }^{\sigma} k_{\delta}\right)$ induces the Connes-Moscovici characteristic homomorphism (2.23).

We will use the cup product (2.28) to untwist the twisted cyclic cohomology. To this end, we first note that the Hopf-cyclic cohomology of the Hopf algebra of Laurent polynomials $k\left[\sigma, \sigma^{-1}\right]$ with coefficients in the SAYD module corresponding to the MPI $\left(\varepsilon, \sigma^{-1}\right)$, is concentrated in degree 1 . More precisely,

$$
H C^{1}\left(k\left[\sigma, \sigma^{-1}\right],{ }^{-1} k\right)=\left\langle\mathbf{1} \otimes\left(1-\sigma^{-1}\right)\right\rangle,
$$

Then specializing (2.28) to

$$
\cup: H C_{\sigma}^{p}(\mathcal{A}) \otimes H C^{1}\left(k\left[\sigma, \sigma^{-1}\right],{ }^{-1} k\right) \rightarrow H C^{p+1}(\mathcal{A}),
$$

we get a characteristic map

$$
\chi: H C_{\sigma}^{n}(\mathcal{A}) \rightarrow H C^{n+1}(\mathcal{A})
$$


which is given in the level of Hochschild cohomology by

$$
\chi(\varphi)\left(a^{0}, \ldots, a^{n+1}\right)=\varphi\left(a^{0}\left(1-\sigma^{-1}\right)\left(a^{1}\right), a^{2}, \ldots, a^{n+1}\right),
$$

and in the level of cyclic cohomology by

$$
\chi(\varphi)\left(a^{0}, \ldots, a^{n+1}\right)=\sum_{\mu \in \operatorname{Sh}(1, n)}(-1)^{\mu} d_{\bar{\mu}(1)} \varphi\left(\partial_{\bar{\mu}(n+1)} \ldots \partial_{\bar{\mu}(2)}\left(1-\sigma^{-1}\right)\left(a^{0}, \ldots, a^{p+q}\right)\right)
$$

We would like to note here that the untwisting phenomenon via a cup product explains in part the dimension drop phenomenon observed in [12], and also Goodman and Krähmer's result [13, Theorem 1.1] that the smash product of a twisted Calabi-Yau algebra of dimension $d$ with the Laurent polynomial ring is an untwisted Calabi-Yau algebra of dimension $d+1$.

\subsection{The characteristic map for compact groups}

We conclude this section by investigating the characteristic homomorphism (2.23) following [6] (see also [17]) in the case of $\mathcal{H}=U(\mathfrak{g})$ and $\mathcal{A}=\mathcal{O}(G)$ where $G$ is one of the (unimodular) groups $S L(N), S O(N)$ or $S p(N)$ where we have a nontrivial invariant Haar functional, and $\mathfrak{g}$ the Lie algebra of $G$. In these cases, $(\varepsilon, 1)$ is a MPI for the Hopf algebra $U(\mathfrak{g})$, and $\mathcal{O}(G)$ is a left $U(\mathfrak{g})$-module algebra via $u(f)(x):=f(x \triangleleft u)$ induced from the action of $\mathfrak{g}$ on $G$ for any $u \in U(\mathfrak{g})$, any $f \in \mathcal{O}(G)$, and any $x \in G$.

Let $\mu$ be the Haar measure on $G$. Then the functional $h: \mathcal{O}(G) \rightarrow k$ defined by $h(f):=\int_{G} f(x) d \mu(x)$ form an invariant trace for the (commutative) Hopf algebra $\mathcal{O}(G)$. Indeed, for any $X \in \mathfrak{g}$, and any $f \in \mathcal{O}(G)$,

$$
\begin{aligned}
h(X \triangleright f) & =\int_{G}(X \triangleright f)(x) d \mu(x) \\
& =\left.\int_{G} \frac{d}{d t}\right|_{t=0} f(\exp (t X) x \exp (t X)) d \mu(x) \\
& =\left.\int_{G} \frac{d}{d t}\right|_{t=0} f(y) d \mu(\exp (t X) y \exp (t X)) \\
& =\left.\int_{G} \frac{d}{d t}\right|_{t=0} f(y) d \mu(y) \\
& =0=\delta(X) h(f),
\end{aligned}
$$

where on the third equality we use the invariance of the Haar measure. We also note that by the unimodularity of the group $G$, the trace of the adjoint representation of $\mathfrak{g}$ on itself vanishes. As a result, we have $\chi: H C^{*}\left(U(\mathfrak{g}), k_{\delta}\right) \rightarrow H C^{*}(\mathcal{O}(G))$ defined as 


$$
\chi\left(u^{1}, \ldots, u^{n}\right)\left(f^{0}, \ldots, f^{n}\right):=\int_{G} f^{0}(x)\left(u^{1}\left(f^{1}\right)\right)(x) \ldots\left(u^{n}\left(f^{n}\right)\right)(x) d \mu(x)
$$

where $u(f) \in \mathcal{O}(G)$, for an arbitrary $u \in U(\mathfrak{g})$ and $f \in \mathcal{O}(G)$, denotes the left coregular action. We also recall from [7, Theorem 15] that

$$
H P^{*}\left(U(\mathfrak{g}), k_{\delta}\right) \cong \bigoplus_{n=* \bmod 2} H_{n}\left(\mathfrak{g}, k_{\delta}\right)
$$

via the anti-symmetrization ant $: k_{\delta} \otimes \bigwedge^{*} \mathfrak{g} \rightarrow C^{*}\left(U(\mathfrak{g}), k_{\delta}\right)$, and from [4, Theorem 46] that

$$
H P^{*}(\mathcal{O}(G)) \cong \bigoplus_{n=* \bmod 2} H_{n}^{\mathrm{dR}}(G)
$$

via a map $\varphi \mapsto C$ given by

$$
\left\langle C, f^{0} d f^{1} \wedge \cdots \wedge d f^{n}\right\rangle=\sum_{\sigma \in S_{n}}(-1)^{\sigma} \varphi\left(f^{0}, f^{\sigma(1)}, \ldots, f^{\sigma(n)}\right)
$$

Here $H_{*}^{\mathrm{dR}}(G)$ refers to the de Rham homology of $G$. In the reverse direction, from [5, Theorem 3.2.14] we have $\Phi: H_{*}^{\mathrm{dR}}(G) \rightarrow H C^{*}(\mathcal{O}(G))$ given by

$$
\Phi(C)\left(f^{0}, f^{1}, \ldots, f^{n}\right)=\left\langle C, f^{0} d f^{1} \wedge \cdots \wedge d f^{n}\right\rangle
$$

Hence, following [6, Lemma 8, 9], we arrive at the commutative diagram

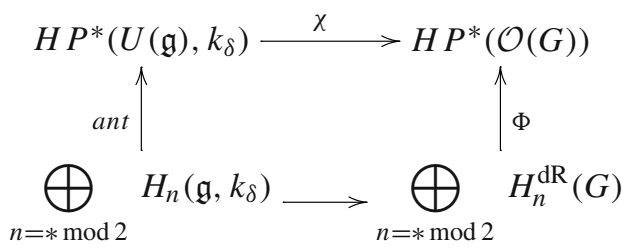

which is the periodic version of (2.32) up to Poincare duality.

\section{Quantum characteristic map}

In this section we will define a quantum analogue of the characteristic homomorphism for compact quantum group algebras. To this end we will first recall the quantum enveloping algebras, and their Hopf-cyclic cohomology, as well as the compact quantum group algebras from [25]. Then using the modular property of the Haar functional we construct a characteristic homomorphism similar to that of Connes and Moscovici $[6]$. 


\subsection{Quantum enveloping algebras (QUE algebras)}

Following [25, Sect. 6.1.2], let $\mathfrak{g}$ be a finite dimensional complex semi-simple Lie algebra, $A=\left[a_{i j}\right]$ the Cartan matrix of $\mathfrak{g}$, and $d_{i} \in\{1,2,3\}$ for $1 \leqslant i \leqslant \ell$ so that $D A=\left[d_{i} a_{i j}\right]$ is the symmetrized Cartan matrix. Let also $q$ be a fixed nonzero complex number such that $q_{i}^{2} \neq 1$, where $q_{i}:=q^{d_{i}}$.

Then the quantum enveloping algebra $U_{q}(\mathfrak{g})$ is the Hopf algebra with $4 \ell$ generators $E_{i}, F_{i}, K_{i}, K_{i}^{-1}, 1 \leqslant i \leqslant \ell$, and the relations

$$
\begin{aligned}
& K_{i} K_{j}=K_{j} K_{i}, \quad K_{i} K_{i}^{-1}=K_{i}^{-1} K_{i}=1, \\
& K_{i} E_{j} K_{i}^{-1}=q_{i}^{a_{i j}} E_{j}, \quad K_{i} F_{j} K_{i}^{-1}=q_{i}^{-a_{i j}} F_{j}, \\
& E_{i} F_{j}-F_{j} E_{i}=\delta_{i j} \frac{K_{i}-K_{i}^{-1}}{q_{i}-q_{i}^{-1}}, \\
& \sum_{r=0}^{1-a_{i j}}(-1)^{r}\left[\begin{array}{c}
1-a_{i j} \\
r
\end{array}\right]_{q_{i}} E_{i}^{1-a_{i j}-r} E_{j} E_{i}^{r}=0, \quad i \neq j, \\
& 1-a_{i j}(-1)^{r}\left[\begin{array}{c}
1-a_{i j} \\
r
\end{array}\right]_{q_{i}} F_{i}^{1-a_{i j}-r} F_{j} F_{i}^{r}=0, \quad i \neq j,
\end{aligned}
$$

where

$$
\left[\begin{array}{l}
n \\
r
\end{array}\right]_{q}=\frac{(n)_{q} !}{(r)_{q} !(n-r)_{q} !}, \quad(n)_{q}:=\frac{q^{n}-q^{-n}}{q-q^{-1}}
$$

The rest of the Hopf algebra structure of $U_{q}(\mathfrak{g})$ is given by

$$
\begin{aligned}
& \Delta\left(K_{i}\right)=K_{i} \otimes K_{i}, \quad \Delta\left(K_{i}^{-1}\right)=K_{i}^{-1} \otimes K_{i}^{-1} \\
& \Delta\left(E_{i}\right)=E_{i} \otimes K_{i}+1 \otimes E_{i}, \quad \Delta\left(F_{j}\right)=F_{j} \otimes 1+K_{j}^{-1} \otimes F_{j} \\
& \varepsilon\left(K_{i}\right)=1, \quad \varepsilon\left(E_{i}\right)=\varepsilon\left(F_{i}\right)=0 \\
& S\left(K_{i}\right)=K_{i}^{-1}, \quad S\left(E_{i}\right)=-E_{i} K_{i}^{-1}, \quad S\left(F_{i}\right)=-K_{i} F_{i} .
\end{aligned}
$$

\subsection{Cohomology of QUE algebras}

Let us recall the Hochschild cohomology of the quantized enveloping algebras $U_{q}(\mathfrak{g})$ from [22]. However, we develop here a different strategy than op.cit.

A modular pair in involution (MPI) for the Hopf algebra $U_{q}(\mathfrak{g})$ is given by [25, Proposition 6.1.6]. Let $K_{\lambda}:=K_{1}^{n_{1}} \ldots K_{\ell}^{n_{\ell}}$ for any $\lambda=\sum_{i} n_{i} \alpha_{i}$, where $n_{i} \in \mathbb{Z}$. Then, $\rho \in \mathfrak{h}^{*}$ being the half-sum of the positive roots of $\mathfrak{g}$, by [25, Proposition 6.1.6] we have

$$
S^{2}(a)=K_{2 \rho} a K_{2 \rho}^{-1}
$$


for all $a \in U_{q}(\mathfrak{g})$. Thus, $\left(\varepsilon, K_{2 \rho}\right)$ is a MPI for the Hopf algebra $U_{q}(\mathfrak{g})$. We shall use the notation $\sigma:=K_{2 \rho}$. In view of the arguments in Sect. 2.2, and following [22], for

$$
U_{q}\left(\mathfrak{b}_{+}\right)=\operatorname{Span}\left\{E_{1}^{r_{1}} \ldots E_{\ell}^{r_{\ell}} K_{1}^{q_{1}} \ldots K_{\ell}^{q_{\ell}} \mid r_{1}, \ldots, r_{\ell} \geqslant 0, q_{1}, \ldots, q_{\ell} \in \mathbb{Z}\right\}
$$

we consider the coextension $\pi: U_{q}(\mathfrak{g}) \rightarrow U_{q}\left(\mathfrak{b}_{+}\right)$which is defined as

$$
\begin{aligned}
\pi & \left(E_{1}^{r_{1}} \cdots E_{\ell}^{r_{\ell}} K_{1}^{q_{1}} \cdots K_{\ell}^{q_{\ell}} F_{1}^{s_{1}} \cdots F_{\ell}^{s_{\ell}}\right) \\
& = \begin{cases}E_{1}^{r_{1}} \cdots E_{\ell}^{r_{\ell}} K_{1}^{q_{1}} \cdots K_{\ell}^{q_{\ell}} & \text { if } r_{1}+\cdots+r_{\ell}=0, \\
0 & \text { otherwise. }\end{cases}
\end{aligned}
$$

Because we have a Poincaré-Birkhoff-Witt basis for $U_{q}(\mathfrak{g})$, it is coflat over the coalgebra $U_{q}\left(\mathfrak{b}_{+}\right)$. Thus, by [22, Proposition 4.8] and [8, Lemma 5.1],

$$
H H^{n}\left(U_{q}(\mathfrak{g}),{ }^{\sigma} k\right)= \begin{cases}k^{\oplus 2^{\ell}} & \text { if } n=\ell \\ 0 & \text { if } n \neq \ell .\end{cases}
$$

In particular, for $\mathfrak{g}=s \ell_{2}$, we calculate the same classes as [8, Proposition 5.9]. Namely,

$$
H H^{n}\left(U_{q}\left(s \ell_{2}\right),{ }^{\sigma} k\right)= \begin{cases}\langle E, K F\rangle & \text { if } n=1 \\ 0 & \text { if } n \neq 1 .\end{cases}
$$

We finally note that along the way to compute the Hochschild (co)homology of $U_{q}(\mathfrak{g})$, regarded as an algebra, the Tor-groups $\operatorname{Tor}_{*}^{U_{q}(\mathfrak{g})}(k, k)$ and the Ext-groups $\operatorname{Ext}_{U_{q}(\mathfrak{g})}^{*}(k, k)$ are obtained in [10].

\subsection{Compact quantum group algebras (CQG algebras)}

In this subsection we will construct a characteristic map $H C^{p}\left(U_{q}(\mathfrak{g}),{ }^{\sigma} k\right) \rightarrow$ $H C^{p+1}\left(\mathcal{O}\left(G_{q}\right)\right)$. In order to do this, we will use the existence of a unique Haar state on the coordinate algebras, as well as their pairing with the QUE algebras.

We begin with the definition of the coordinate algebras of the quantum groups from [25, Sect. 11.3].

Definition 3.1 A Hopf $*$-algebra $\mathcal{H}$ is called a compact quantum group (CQG) algebra if $\mathcal{H}$ is the linear span of all matrix elements of finite dimensional unitary corepresentations of $\mathcal{H}$. A compact matrix quantum group (CMQG) algebra is a CQG algebra which is generated, as an algebra, by finitely many elements.

Among examples of CMQG algebras are the Hopf $*$-algebras $\mathcal{O}\left(U_{q}(N)\right), \mathcal{O}\left(S U_{q}(N)\right)$, $\mathcal{O}\left(O_{q}(N ; \mathbb{R})\right), \mathcal{O}\left(S O_{q}(N ; \mathbb{R})\right)$ and $\mathcal{O}\left(S p_{q}(N)\right)$, see [25, Example 11.7]. For any compact group $G$, the Hopf algebra $R(G)$ of representative functions is a CQG algebra. Also, if $\mu$ is the Haar measure on such a group $G$ then $h: R(G) \rightarrow k$ given by $h(f):=\int_{G} f(x) d \mu(x)$ is the corresponding Haar functional. 
Theorem 3.2 If $\mathcal{A}=\mathcal{O}\left(G_{q}\right)$ is a CQG algebra, then there is a characteristic map of the form

$$
\begin{aligned}
& \chi_{q}: H C^{*}\left(U_{q}(\mathfrak{g}),{ }^{\sigma} k\right) \rightarrow H C_{\sigma^{-1}}^{*}\left(\mathcal{O}\left(G_{q}\right)\right), \\
& \chi_{q}\left(y^{1}, \ldots, y^{n}\right)\left(f^{0}, \ldots, f^{n}\right):=h\left(f^{0} y^{1}\left(f^{1}\right) \ldots y^{n}\left(f^{n}\right)\right) .
\end{aligned}
$$

Proof Every CQG algebra possesses a unique (left and right invariant) Haar functional due to their cosemisimplicity [25, Theorem 11.13]. In view of [25, Eq. 11(36)], it follows from [25, Proposition 11.34] that the Haar functional $h: \mathcal{A} \rightarrow k$ on a CQG algebra of the form $\mathcal{A}=\mathcal{O}\left(G_{q}\right)$ has the crucial property that

$$
h(a b)=h(b(\sigma \triangleright a \triangleleft \sigma))
$$

for $\sigma=K_{2 \rho} \in U_{q}(\mathfrak{g})$ with the left ad the right coregular actions. We will observe the compatibility of the map (3.5) with the Hopf-cyclic coface operators (2.17), codegeneracies (2.18), and the cyclic operator (2.19). Accordingly, we first show that

$$
\begin{aligned}
\chi_{q}\left(d_{0}\left(y^{1}, \ldots, y^{n}\right)\right)\left(f^{0}, \ldots, f^{n+1}\right) & =\chi_{q}\left(1, y^{1}, \ldots, y^{n}\right)\left(f^{0}, \ldots, f^{n+1}\right) \\
& =h\left(f^{0} f^{1} y^{1}\left(f^{2}\right) \ldots y^{n}\left(f^{n+1}\right)\right) \\
& =d_{0} \chi_{q}\left(y^{1}, \ldots, y^{n}\right)\left(f^{0}, \ldots, f^{n+1}\right) .
\end{aligned}
$$

Next we observe for $1 \leqslant i \leqslant n$ that

$$
\begin{aligned}
\chi_{q}\left(d_{i}\left(y^{1}, \ldots, y^{n}\right)\right)\left(f^{0}, \ldots, f^{n+1}\right) & =\chi_{q}\left(y^{1}, \ldots, \Delta\left(y^{i}\right), \ldots, y^{n}\right)\left(f^{0}, \ldots, f^{n+1}\right) \\
& =h\left(f^{0} y^{1}\left(f^{1}\right) \ldots y^{i}\left(f^{i} f^{i+1}\right) \ldots y^{n}\left(f^{n+1}\right)\right) \\
& =d_{i} \chi_{q}\left(y^{1}, \ldots, y^{n}\right)\left(f^{0}, \ldots, f^{n+1}\right) .
\end{aligned}
$$

As for the last coface map we have

$$
\begin{aligned}
\chi_{q}\left(d_{n+1}\left(y^{1}, \ldots, y^{n}\right)\right)\left(f^{0}, \ldots, f^{n+1}\right) & =\chi_{q}\left(y^{1}, \ldots, y^{n}, \sigma\right)\left(f^{0}, \ldots, f^{n+1}\right) \\
& =h\left(f^{0} y^{1}\left(f^{1}\right) \ldots y^{n}\left(f^{n}\right) \sigma\left(f^{n+1}\right)\right) \\
& =h\left(\left(f^{n+1} \triangleleft \sigma^{-1}\right) f^{0} y^{1}\left(f^{1}\right) \ldots y^{n}\left(f^{n}\right)\right) \\
& =\chi_{q}\left(y^{1}, \ldots, y^{n}\right)\left(\left(f^{n+1} \triangleleft \sigma^{-1}\right) f^{0}, \ldots, f^{n}\right) \\
& =d_{n+1} \chi_{q}\left(y^{1}, \ldots, y^{n}\right)\left(f^{0}, \ldots, f^{n+1}\right) .
\end{aligned}
$$

We proceed to the codegeneracies. We have,

$$
\begin{aligned}
\chi_{q}\left(s_{j}\left(y^{1}, \ldots, y^{n}\right)\right)\left(f^{0}, \ldots, f^{n-1}\right) & =\chi_{q}\left(y^{1}, \ldots, \varepsilon\left(y^{j}\right), \ldots, y^{n}\right)\left(f^{0}, \ldots, f^{n-1}\right) \\
& =\varepsilon\left(y^{j}\right) h\left(f^{0} y^{1}\left(f^{1}\right) \ldots y^{j-1}\left(f^{j-1}\right) y^{j+1}\left(f^{j+1}\right) \ldots y^{n}\left(f^{n-1}\right)\right) \\
& =\chi_{q}\left(y^{1}, \ldots, y^{n}\right)\left(f^{0}, \ldots, f^{j}, 1, f^{j+1}, \ldots f^{n-1}\right) \\
& =s_{j} \chi_{q}\left(y^{1}, \ldots, y^{n}\right)\left(f^{0}, \ldots, f^{n-1}\right) .
\end{aligned}
$$


Finally, we consider the compatibility with the cyclic operator. We have

$$
\begin{aligned}
\chi_{q}\left(t\left(y^{1}, \ldots, y^{n}\right)\right)\left(f^{0}, \ldots, f^{n+1}\right) & =\chi_{q}\left(S\left(y^{1}\right)\left(y^{2}, \ldots, y^{n}, \sigma\right)\right)\left(f^{0}, \ldots, f^{n}\right) \\
& =h\left(f^{0} S\left(y^{1}\right)\left(y^{2}, \ldots, y^{n}, \sigma\right)\left(f^{1}, \ldots, f^{n}\right)\right) \\
& =h\left(y_{(1)}^{1}\left(f^{0} S\left(y_{(2)}^{1}\right)\left(y^{2}, \ldots, y^{n}, \sigma\right)\left(f^{1}, \ldots, f^{n}\right)\right)\right) \\
& =h\left(y^{1}\left(f^{0}\right) y^{2}\left(f^{1}\right) \ldots y^{n}\left(f^{n-1}\right) \sigma\left(f^{n}\right)\right) \\
& =h\left(\left(f^{n} \triangleleft \sigma^{-1}\right) y^{1}\left(f^{0}\right) y^{2}\left(f^{1}\right) \ldots y^{n}\left(f^{n-1}\right)\right) \\
& =\chi_{q}\left(y^{1}, \ldots, y^{n}\right)\left(\left(f^{n+1} \triangleleft \sigma^{-1}\right), f^{0}, \ldots, f^{n}\right) \\
& =t \chi_{q}\left(y^{1}, \ldots, y^{n}\right)\left(f^{0}, \ldots, f^{n+1}\right) .
\end{aligned}
$$

As a result, for a compact group $G$ with Lie algebra $\mathfrak{g}$, the morphism $\chi_{q}$ defined on the chain level by (3.5) induces a morphism $H C^{*}\left(U_{q}(\mathfrak{g}),{ }^{\sigma} k\right) \rightarrow H C_{\sigma^{-1}}^{*}\left(\mathcal{O}\left(G_{q}\right)\right)$ in the level of cohomology.

Remark 3.3 We would like remark that the modularity (3.6) of the Haar functional is not given by a module algebra action of $U_{q}(\mathfrak{g})$ on $\mathcal{O}\left(G_{q}\right)$. However, it is observed in $[28$, Theorems 1,2$]$ that it can be viewed as the module algebra action of the modular square of $U_{q}(\mathfrak{g})$, see [27, Example 3.14]. Then the same Haar functional induces a Connes-Moscovici characteristic map whose target is now the ordinary cyclic cohomology of $\mathcal{O}\left(G_{q}\right)$, [28, Theorem 2], which is observed to be zero for $G=S U(2),[28$, Theorem 9]. On the other hand, the characteristic map (3.5) is also given by [27, Theorem 8.2].

From Sect. 2.9 we conclude the following.

Corollary 3.4 If $\mathcal{A}=\mathcal{O}\left(G_{q}\right)$ is a CQG algebra, then there is a characteristic map of the form

$$
\tilde{\chi}_{q}: H C^{n}\left(U_{q}(\mathfrak{g}),{ }^{\sigma} k\right) \rightarrow H C^{n+1}\left(\mathcal{O}\left(G_{q}\right)\right)
$$

for every $n \geqslant 0$.

\section{The characteristic map between $U_{q}\left(s \ell_{2}\right)$ and $\mathcal{O}\left(S L_{q}(2)\right)$}

In this section we show the non-triviality of the characteristic map between cohomologies of $U_{q}\left(s \ell_{2}\right)$ and $\mathcal{O}\left(S L_{q}(2)\right)$. We compare the classes we obtain in its image by the classes computed in [30].

\subsection{The coordinate algebra $\mathcal{O}\left(S L_{q}(2)\right)$}

Let us begin with the definition of the coordinate algebra $\mathcal{O}\left(S L_{q}(2)\right)$ of the quantum group $S L_{q}(2)$. By [25, Sect. 4.1.2], it is the algebra generated by

$$
\mathbf{t}=\left(\begin{array}{ll}
a & b \\
c & d
\end{array}\right)
$$


subject to the relations

$$
\begin{aligned}
& a b=q b a, \quad a c=q c a, \quad a d=d a+\left(q-q^{-1}\right) b c \\
& b c=c b, \quad b d=q d b, \quad c d=q d c, \quad a d-q b c=1 .
\end{aligned}
$$

The rest of the Hopf algebra structure is given by

$$
\Delta(\mathbf{t})=\mathbf{t} \otimes \mathbf{t}, \quad \varepsilon(\mathbf{t})=1, \quad S(\mathbf{t})=\left(\begin{array}{cc}
d & -q^{-1} b \\
-q c & a
\end{array}\right)
$$

Moreover, it is proved in [25, Theorem 4.21] that

$$
\langle K, a\rangle=q^{-1}, \quad\langle K, d\rangle=q, \quad\langle E, c\rangle=\langle F, b\rangle=1
$$

determines a non-degenerate pairing between the Hopf algebras $U_{q}\left(s \ell_{2}\right)$ and $\mathcal{O}\left(S L_{q}(2)\right)$.

\subsection{The quantum characteristic map}

It is shown in [25, Theorem 4.14] that there exists a unique invariant linear functional $h: \mathcal{O}\left(S L_{q}(2)\right) \rightarrow k$ such that $h(1)=1$. This is the Haar functional of $\mathcal{O}\left(S L_{q}(2)\right)$ as defined in Sect. 3.3. This functional satisfies

$$
((\operatorname{Id} \otimes h) \circ \Delta)(x)=h(x) 1=((h \otimes \operatorname{Id}) \circ \Delta)(x)
$$

for all $x \in \mathcal{O}\left(S L_{q}(2)\right)$. More explicitly, by [25, Theorem 4.14],

$$
h\left(a^{r} b^{k} c^{\ell}\right)=h\left(b^{k} c^{\ell} d^{r}\right)= \begin{cases}0, & r \neq 0, \text { or } k \neq \ell \\ (-1)^{k} \frac{q-q^{-1}}{q^{k+1}-q^{-(k+1)}}, & r=0, \text { and } k=\ell .\end{cases}
$$

Furthermore, by [25, Proposition 4.15] the Haar functional $h: \mathcal{O}\left(S L_{q}(2)\right) \rightarrow k$ is not central (a trace), instead

$$
h(x y)=h(\vartheta(y) x)
$$

for all $x, y \in \mathcal{O}\left(S L_{q}(2)\right)$ where $\vartheta: \mathcal{O}\left(S L_{q}(2)\right) \rightarrow \mathcal{O}\left(S L_{q}(2)\right)$ is the automorphism given by [25, Proposition 4.5] as

$$
\vartheta(a)=q^{2} a, \quad \vartheta(b)=b, \quad \vartheta(c)=c, \quad \vartheta(d)=q^{-2} d .
$$

Lemma 4.1 The automorphism $\vartheta: \mathcal{O}\left(S L_{q}(2)\right) \rightarrow \mathcal{O}\left(S L_{q}(2)\right)$ can be given by the action of $K^{-1} \in U_{q}\left(s \ell_{2}\right)$ in the sense that $\vartheta(x)=K^{-1} \triangleright x \triangleleft K^{-1}$ for any $x \in \mathcal{O}\left(S L_{q}(2)\right)$. 
Proof In view of the pairing (4.4) we have

$$
\begin{aligned}
K^{-1} \triangleright a \triangleleft K^{-1}= & \left\langle K, S\left(a_{(1)}\right)\right\rangle a_{(2)}\left\langle K, S\left(a_{(3)}\right)\right\rangle \\
= & \langle K, S(a)\rangle a\langle K, S(a)\rangle+\langle K, S(b)\rangle c\langle K, S(a)\rangle \\
& +\langle K, S(a)\rangle b\langle K, S(c)\rangle+\langle K, S(b)\rangle d\langle K, S(c)\rangle \\
= & \langle K, d\rangle^{2} a=q^{2} a=\vartheta(a) .
\end{aligned}
$$

Similarly, we have

$$
\begin{aligned}
K^{-1} \triangleright b \triangleleft K^{-1}= & \left\langle K, S\left(b_{(1)}\right)\right\rangle b_{(2)}\left\langle K, S\left(b_{(3)}\right)\right\rangle \\
= & \langle K, S(a)\rangle a\langle K, S(b)\rangle+\langle K, S(b)\rangle c\langle K, S(b)\rangle \\
& +\langle K, S(a)\rangle b\langle K, S(d)\rangle+\langle K, S(b)\rangle d\langle K, S(d)\rangle \\
= & \langle K, d\rangle\langle K, a\rangle b=b=\vartheta(b),
\end{aligned}
$$

and

$$
\begin{aligned}
K^{-1} \triangleright c \triangleleft K^{-1}= & \left\langle K, S\left(c_{(1)}\right)\right\rangle c_{(2)}\left\langle K, S\left(c_{(3)}\right)\right\rangle \\
= & \langle K, S(c)\rangle a\langle K, S(a)\rangle+\langle K, S(d)\rangle c\langle K, S(a)\rangle \\
& +\langle K, S(c)\rangle b\langle K, S(c)\rangle+\langle K, S(d)\rangle d\langle K, S(c)\rangle \\
= & \langle K, a\rangle\langle K, d\rangle c=c=\vartheta(c),
\end{aligned}
$$

and finally

$$
\begin{aligned}
K^{-1} \triangleright d \triangleleft K^{-1}= & \left\langle K, S\left(d_{(1)}\right)\right\rangle d_{(2)}\left\langle K, S\left(d_{(3)}\right)\right\rangle \\
= & \langle K, S(c)\rangle a\langle K, S(b)\rangle+\langle K, S(d)\rangle c\langle K, S(b)\rangle \\
& +\langle K, S(c)\rangle b\langle K, S(d)\rangle+\langle K, S(d)\rangle d\langle K, S(d)\rangle \\
= & \langle K, a\rangle^{2} d=q^{-2} d=\vartheta(d) .
\end{aligned}
$$

as we wanted to show.

Lemma 4.2 The right coregular action of $\sigma^{-1}=K^{-1} \in U_{q}\left(s \ell_{2}\right)$ on $\mathcal{O}\left(S L_{q}(2)\right)$ is an automorphism of $\mathcal{O}\left(S L_{q}(2)\right)$.

Proof We have

$$
\begin{aligned}
(f g) \triangleleft \sigma^{-1} & =\left\langle(f g)_{(1)}, \sigma^{-1}\right\rangle(f g)_{(2)} \\
& =\left\langle f \partial . s 1 g_{(1)}, \sigma^{-1}\right\rangle f_{(2)} g_{(2)} \\
& =\left\langle f_{(1)}, \sigma^{-1}\right\rangle\left\langle g_{(1)}, \sigma^{-1}\right\rangle f_{(2)} g_{(2)} \\
& =\left(f \triangleleft \sigma^{-1}\right)\left(g \triangleleft \sigma^{-1}\right) .
\end{aligned}
$$


Lemma 4.3 The Haar functional $h: \mathcal{O}\left(S L_{q}(2)\right) \rightarrow k$ is $\varepsilon$-invariant with respect to the left coregular action of $U_{q}\left(s \ell_{2}\right)$ on $\mathcal{O}\left(S L_{q}(2)\right)$.

Proof Via the invariance of (4.5), for any $y \in U_{q}\left(s \ell_{2}\right)$ and $f \in \mathcal{O}\left(S L_{q}(2)\right)$ we have

$$
h(y(f))=h\left(f_{(1)}\right)\left\langle y, f_{(2)}\right\rangle=h(f)\langle y, 1\rangle=\varepsilon(y) h(f) .
$$

As a result, using Theorem 3.2 we get the following.

Corollary 4.4 For the Hopf algebra $U_{q}\left(s \ell_{2}\right)$ with the modular pair $(\varepsilon, \sigma)$ in involution, the Haar functional $h: \mathcal{O}\left(S L_{q}(2)\right) \rightarrow k$ determines a characteristic homomorphism

$$
\begin{aligned}
& \chi_{q}: H C^{*}\left(U_{q}\left(s \ell_{2}\right),{ }^{\sigma} k\right) \rightarrow H C_{\sigma^{-1}}^{*}\left(\mathcal{O}\left(S L_{q}(2)\right)\right), \\
& \chi_{q}\left(y^{1}, \ldots, y^{n}\right)\left(f^{0}, \ldots, f^{n}\right):=h\left(f^{0} y^{1}\left(f^{1}\right) \ldots y^{n}\left(f^{n}\right)\right),
\end{aligned}
$$

where for any $x, y \in U_{q}\left(s \ell_{2}\right)$ and $f \in \mathcal{O}\left(S L_{q}(2)\right), y(f)(x):=f(x y)$ is the left coregular action.

Combining with Corollary 3.4, we obtain the following result.

Corollary 4.5 For the Hopf algebra $U_{q}\left(s \ell_{2}\right)$ with the modular pair $(\varepsilon, \sigma)$ in involution, the Haar functional $h: \mathcal{O}\left(S L_{q}(2)\right) \rightarrow k$ determines a characteristic homomorphism

$$
\widetilde{\chi}_{q}: H C^{*}\left(U_{q}\left(s \ell_{2}\right),{ }^{\sigma} k\right) \rightarrow H C^{*+1}\left(\mathcal{O}\left(S L_{q}(2)\right)\right)
$$

\subsection{The non-triviality of the quantum characteristic map}

In order to discuss the non-triviality of the characteristic homomorphism (4.9) we recall the results of [30]. First define

$$
d(t):=\left\{\begin{array}{cl}
d^{t} & \text { if } t \geqslant 0 \\
a^{-t} & \text { if } t<0
\end{array}\right.
$$

and

$$
(x ; q)_{n}:=(1-x)(1-q x) \cdots\left(1-q^{n-1} x\right) .
$$

In [30] it is calculated that

$$
H C^{n}\left(\mathcal{O}\left(S L_{q}(2)\right)\right)= \begin{cases}k\left[\tau_{\text {even }}\right] \oplus \bigoplus_{i, j, k, \ell>0} k\left[\tau_{a}^{i}\right] \oplus k\left[\tau_{b}^{j}\right] \oplus k\left[\tau_{c}^{k}\right] \oplus k\left[\tau_{d}^{\ell}\right] & \text { if } n=0 \\ k S^{\lfloor n / 2\rfloor}\left[\tau_{\text {even }}\right] & \text { if } n>0 \text { is even } \\ k S^{\lfloor n / 2\rfloor}\left[\tau_{\text {odd }}\right] & \text { if } n \text { is odd }\end{cases}
$$


where

$$
\begin{aligned}
\tau_{a}^{l}\left(d(t) b^{m} c^{n}\right) & =\delta_{t,-l} \delta_{m, 0} \delta_{n, 0}, \\
\tau_{b}^{l}\left(d(t) b^{m} c^{n}\right) & =\delta_{t, 0} \delta_{m-n, l} \frac{q^{l}-1}{q^{l+2 n}-1}(-q)^{n}, \\
\tau_{c}^{l}\left(d(t) b^{m} c^{n}\right) & =\delta_{t, 0} \delta_{n-m, l} \frac{q^{l}-1}{q^{l+2 m}-1}(-q)^{m}, \\
\tau_{d}^{l}\left(d(t) b^{m} c^{n}\right) & =\delta_{t, l} \delta_{m, 0} \delta_{n, 0}, \\
\tau_{\text {even }}\left(d(t) b^{m} c^{n}\right) & =\delta_{t, 0} \delta_{m, 0} \delta_{n, 0},
\end{aligned}
$$

and finally

$$
\begin{aligned}
\tau_{\text {odd }}\left(d(t) b^{m} c^{n}, d(\widetilde{t}) b^{\widetilde{m}} c^{\widetilde{n}}\right) & =0 \quad \text { if } t+\widetilde{t} \neq 0 \\
\tau_{\text {odd }}\left(a^{t} b^{m} c^{n}, d^{\tilde{t}} b^{\widetilde{m}} c^{\widetilde{n}}\right) & =\tau_{\text {odd }}\left(d^{t} b^{m} c^{n}, a^{\widetilde{t}} b^{\widetilde{m}} c^{\widetilde{n}}\right) \\
& =(n-m)(-q)^{n+\widetilde{n}} q^{t(\widetilde{m}+\widetilde{n})} \frac{\left(q^{2} ; q^{2}\right)_{t}}{\left(q^{2(n+\widetilde{n})} ; q^{2}\right)_{t+1}} \delta_{t, \widetilde{t}} \delta_{m+\widetilde{m}, n+\widetilde{n}} .
\end{aligned}
$$

We are now ready to compute the images, under the characteristic homomorphism (4.10), of the Hopf-cyclic classes (3.4).

Proposition 4.6 The classes $\left[\widetilde{\chi}_{q}(E)\right],\left[\tilde{\chi}_{q}(K F)\right] \in H C^{2}\left(\mathcal{O}\left(S L_{q}(2)\right)\right)$ are nontrivial.

Proof By the definition (4.6) of the Haar functional $h: \mathcal{O}\left(S L_{q}(2)\right) \rightarrow k$, we have

$$
\begin{aligned}
\tilde{\chi}_{q}(E)\left(x_{0}, x_{1}, x_{2}\right)= & -\chi_{q}(E)\left(x_{0}(1-\sigma)\left(x_{1}\right), x_{2}\right)-\chi_{q}(E)\left(x_{0} x_{1},(1-\sigma)\left(x_{2}\right)\right) \\
& +\chi_{q}(E)\left(x_{0},(1-\sigma)\left(x_{1}\right) x_{2}\right) \\
= & h\left(x_{0}(1-\sigma)\left(x_{1}\right) E\left(x_{2}\right)\right)-h\left(x_{0} x_{1} E(1-\sigma)\left(x_{2}\right)\right) \\
& +h\left(x_{0} E\left((1-\sigma)\left(x_{1}\right) x_{2}\right)\right) .
\end{aligned}
$$

We consider the element $\omega=b \otimes c^{2} \otimes a-a \otimes b \otimes c^{2} \in \mathcal{O}\left(S L_{q}(2)\right)^{\otimes 3}$ on which any coboundary vanishes, that is, for any cyclic 1 -cocycle $\varphi: \mathcal{O}\left(S L_{q}(2)\right)^{\otimes 2} \rightarrow k$,

$$
\begin{aligned}
b \varphi(\omega)= & \varphi\left(b c^{2} \otimes a\right)-\varphi\left(b \otimes c^{2} a\right)+\varphi\left(a b \otimes c^{2}\right) \\
& -\varphi\left(a b \otimes c^{2}\right)+\varphi\left(a \otimes b c^{2}\right)-\varphi\left(c^{2} a \otimes b\right)=0 .
\end{aligned}
$$

Hence it follows from

$$
\begin{aligned}
\tilde{\chi}_{q}(E)(\omega) & =h\left(b(1-\sigma)\left(c^{2}\right) E(a)\right)-h\left(b c^{2} E(1-\sigma)(a)\right)+h\left(b E\left((1-\sigma)\left(c^{2}\right) a\right)\right) \\
& =\left(2 q^{-2}+q^{-1}-1\right) h\left(b^{2} c^{2}\right)=\left(2 q^{-2}+q^{-1}-1\right) \frac{q-q^{-1}}{q^{3}-q^{-3}} \neq 0
\end{aligned}
$$


that $\left[\tilde{\chi}_{q}(E)\right] \neq 0$. Using the element $\omega=c \otimes b^{2} \otimes d-d \otimes c \otimes b^{2} \in \mathcal{O}\left(S L_{q}(2)\right)^{\otimes 3}$, we similarly arrive at $\left[\widetilde{\chi}_{q}(K F)\right] \neq 0$.

In view of (4.11) we conclude the following.

Corollary 4.7 We have $\left[\tilde{\chi}_{q}(K F)\right]=\left[\tilde{\chi}_{q}(E)\right]=\left[S\left(\tau_{\text {even }}\right)\right] \in H C^{2}\left(\mathcal{O}\left(S L_{q}(2)\right)\right)$.

\section{The $q$-Index Cocycle for the Standard Podleś Sphere}

In this section we discuss the equivariant generalization of (4.9), and we capture the Schmüdgen-Wagner index cocycle of [34] in the image of the equivariant characteristic map.

\subsection{QUE algebra $U_{q}\left(s u_{2}\right)$ and CQG algebra $\mathcal{O}\left(S U_{q}(2)\right)$}

Let $\mathcal{O}\left(S U_{q}(2)\right)$ be the coordinate Hopf algebra of the compact quantum group $S U_{q}(2)$, see [25, Sect. 4.1.4]. Following the notation of [34], let also $U_{q}\left(s u_{2}\right)$ be the Hopf algebra generated by $E, F, K, K^{-1}$ subject to the relations

$$
K K^{-1}=K^{-1} K=1, \quad K E=q E K, \quad F K=q K F, \quad E F-F E=\frac{K^{2}-K^{-2}}{q-q^{-1}},
$$

whose Hopf algebra structure is given by

$$
\begin{aligned}
& \Delta(K)=K \otimes K, \quad \Delta\left(K^{-1}\right)=K^{-1} \otimes K^{-1}, \\
& \Delta(E)=E \otimes K+K^{-1} \otimes E, \quad \Delta(F)=F \otimes K+K^{-1} \otimes F, \\
& \varepsilon(K)=\varepsilon\left(K^{-1}\right)=1, \quad \varepsilon(E)=\varepsilon(F)=0, \\
& S(K)=K^{-1}, \quad S(E)=-q E, \quad S(F)=-q^{-1} F .
\end{aligned}
$$

We note that, in the terminology of [25], it is the Hopf algebra $\breve{U}_{q}\left(s \ell_{2}\right)$.

We also note that the non-degenerate pairing between the Hopf algebras $U_{q}\left(s u_{2}\right)$ and $\mathcal{O}\left(S U_{q}(2)\right)$ is given by

$$
\left\langle K^{ \pm 1}, d\right\rangle=\left\langle K^{\mp 1}, a\right\rangle=q^{ \pm 1 / 2}, \quad\langle E, c\rangle=\langle F, b\rangle=1 .
$$

Then, $\mathcal{O}\left(S U_{q}(2)\right)$ is a left (and a right) $U_{q}\left(s u_{2}\right)$-module algebra via the coregular action.

\subsection{The standard Podleś Sphere}

The coordinate $*$-algebra $\mathcal{O}\left(S_{q}^{2}\right)$ of the standard Podleś sphere [31] is the unital $*$ algebra with three generators $A=A^{*}, B, B^{*}$ with the relations

$$
B A=q^{2} A B, \quad A B^{*}=q^{2} B^{*} A, \quad B^{*} B=A-A^{2}, \quad B B^{*}=q^{2} A-q^{4} A^{2} .
$$


It is also possible to view it as the $K$-invariant subalgebra

$$
\mathcal{O}\left(S_{q}^{2}\right)=\left\{x \in \mathcal{O}\left(S U_{q}(2)\right) \mid x \triangleleft K=x\right\}
$$

of $\mathcal{O}\left(S U_{q}(2)\right)$. We also recall from [34] that for the Haar state $h$ on $\mathcal{O}\left(S U_{q}(2)\right)$, we have $h(x y)=h\left(\left(K^{-2}(y) \triangleleft K^{-2}\right) x\right)$ for any $x, y \in \mathcal{O}\left(S U_{q}(2)\right)$. Hence, for any $x, y \in \mathcal{O}\left(S_{q}^{2}\right)$ we have $h(x y)=h(\sigma(y) x)$ with $\sigma=K^{-2}$.

\subsection{Equivariant Hopf-cyclic cohomology and its actions}

Let us also recall from [34, Lemma 4.1] that

$$
h\left(R_{F}(x) R_{E}(y)\right)=q^{2} h\left(R_{E}(x) R_{F}(y)\right),
$$

for all $x, y \in \mathcal{O}\left(S_{q}^{2}\right)$. As a result, the functional $\tau: \mathcal{O}\left(S_{q}^{2}\right)^{\otimes 3} \rightarrow k$, defined for all $x, y, z \in \mathcal{O}\left(S_{q}^{2}\right)$ as

$$
\tau(x, y, z):=h\left(x R_{F}(y) R_{E}(z)-q^{2} x R_{E}(y) R_{F}(z)\right),
$$

is a nontrivial $\sigma$-twisted cyclic 2-cocycle, i.e. $[\tau] \in H C_{\sigma}^{2}\left(\mathcal{O}\left(S_{q}^{2}\right)\right)$.

On the other hand, we recall the cup product construction defined in [33, Theorem 3.3]. Let $\mathcal{H}$ be a Hopf algebra, $\mathcal{K} \subseteq \mathcal{H}$ a cocommutative Hopf subalgebra, and finally $V$ and $N$ are SAYD modules over $\mathcal{K}$ and $\mathcal{H}$ respectively. It is proved in [33, Theorem 3.1] that

$$
C_{\mathcal{K}}(\mathcal{H}, V, N):=\bigoplus_{p \geqslant 0} C_{\mathcal{K}}^{p}(\mathcal{H}, V, N), \quad C_{\mathcal{K}}^{p}(\mathcal{H}, V, N):=\operatorname{Hom}_{\mathcal{K}}\left(V, N \otimes_{\mathcal{H}} \mathcal{H}^{\otimes p+1}\right)
$$

is a cocyclic module, computing the equivariant Hopf-cyclic cohomology $H C_{\mathcal{K}}$ $(\mathcal{H}, V, N)$, via

$$
\begin{aligned}
& d_{i}(\phi)(v)= \begin{cases}\partial_{i}(\phi(v)), & \text { if } 0 \leq i \leq p, \\
\partial_{p+1}\left(\phi\left(v_{<0>}\right)\right) \triangleleft S\left(v_{<-1>}\right), & \text { if } i=p+1,\end{cases} \\
& s_{j}(\phi)(v)=\sigma_{j}(\phi(v)), \quad \text { for } 0 \leq j \leq p-1, \\
& t_{p}(\phi)(v)=\tau_{p}\left(\phi\left(v_{<0>}\right)\right) \triangleleft S\left(v_{<-1>}\right),
\end{aligned}
$$

where the morphisms $\partial_{i}, \sigma_{j}$, and $\tau$ are those given by (2.25), (2.26) and (2.27), and

$$
\left(n \otimes_{\mathcal{H}} h^{0} \otimes \cdots \otimes h^{p}\right) \triangleleft u=n \otimes_{\mathcal{H}} h^{0} \otimes \cdots \otimes h^{p} u .
$$

We recall also that $\phi \in C_{\mathcal{K}}^{p}(\mathcal{H}, V, N)$ if

$$
\phi(v \cdot u)=\phi(v) \cdot u,
$$


for any $u \in \mathcal{K}$ where

$$
\left(n \otimes_{\mathcal{H}} h^{0} \otimes \cdots \otimes h^{p}\right) \cdot u:=n \otimes_{\mathcal{H}} h^{0} u_{(1)} \otimes \cdots \otimes h^{p} u_{(p+1)} .
$$

Employing the notation $\phi(v)_{=}=: \phi(v)^{[-1]} \otimes_{\mathcal{H}} \phi(v)^{[0]} \otimes \cdots \otimes \phi(v)^{[p]}$ for $\phi \in$ $C_{\mathcal{K}}^{p}(\mathcal{H}, V, N)$, let us set $\Psi: C_{\mathcal{K}}^{p}(\mathcal{H}, V, N) \otimes C_{\mathcal{H}}^{p}(\mathcal{A}, N) \longrightarrow C_{\mathcal{K}}^{p}(\mathcal{A}, V)$ as

$$
\begin{aligned}
& \Psi(\phi \otimes \psi)\left(v \otimes x_{0} \otimes \cdots \otimes x_{p}\right) \\
& \quad=\psi\left(\phi(v)^{[-1]} \otimes \phi(v)^{[0]}\left(x_{0}\right) \otimes \phi(v)^{[1]}\left(x_{1}\right) \otimes \cdots \otimes \phi(v)^{[p]}\left(x_{p}\right)\right) .
\end{aligned}
$$

Then the equivariant characteristic map is given by the cup product

$$
H C_{\mathcal{K}}^{p}(\mathcal{H}, V, N) \otimes H C_{\mathcal{H}}^{q}(\mathcal{A}, N) \rightarrow H C_{\mathcal{K}}^{p+q}(\mathcal{A}, V), \quad[\phi] \cup[\varphi]:=\Psi(\operatorname{Sh}(\phi \otimes \varphi)) .
$$

using the shuffle map Sh : Tot $\rightarrow$ Diag, from the total of the tensor product of the complexes $C_{\mathcal{K}}^{*}(\mathcal{H}, V, N)$ and $C_{\mathcal{H}}^{*}(\mathcal{A}, N)$ to the diagonal. Adopting the notation of [23], the shuffle map is given by

$$
\text { Sh }: \operatorname{Tot}^{n} \rightarrow \operatorname{Diag}^{n}, \quad \text { Sh }=\sum_{p+q=n} \nabla_{p, q},
$$

where

$$
\nabla_{p, q}=\sum_{\mu \in \mathrm{Sh}_{q, p}}(-1)^{\mu} d_{\bar{\mu}(p+q)} \ldots d_{\bar{\mu}(p+1)} \partial_{\bar{\mu}(p)} \ldots \partial_{\bar{\mu}(1)}
$$

\subsection{The $q$-index cocycle for the standard Podleś sphere}

Let us take $\mathcal{H}=U_{q}\left(s u_{2}\right), \mathcal{K}=k\left[\sigma, \sigma^{-1}\right], N={ }^{\sigma^{-1}} k, V={ }^{\sigma} k$ and $\mathcal{A}=\mathcal{O}\left(S_{q}^{2}\right)$. On the next proposition we compute the $q$-index cocycle in the equivariant Hopf-cyclic cohomology.

Proposition 5.1 Let $F \in C_{\mathcal{K}}^{2}(\mathcal{H}, V, N)$ be given by

$$
F(\mathbf{1}):=\mathbf{1} \otimes_{\mathcal{H}} 1 \otimes\left(K F \otimes E K^{3}-E K \otimes K^{3} F\right)-\left(q^{3}-q\right)^{-1} \mathbf{1} \otimes_{\mathcal{H}} 1 \otimes 1 \otimes K^{4} .
$$

Then, $[F] \in H C_{\mathcal{K}}^{2}(\mathcal{H}, V, N)$, i.e. $F$ is an equivariant cyclic 2-cocycle.

Proof Let us first show that $F$ is indeed $\mathcal{K}$-equivariant. For any $K^{m}$ with $m \in \mathbb{Z}$ we have

$$
\begin{aligned}
F(\mathbf{1}) \cdot K^{m}= & \mathbf{1} \otimes_{\mathcal{H}} K^{m} \otimes\left(K F K^{m} \otimes E K^{3+m}-E K^{m+1} \otimes K^{3} F K^{m}\right) \\
& -\left(q^{3}-q\right)^{-1} \mathbf{1} \otimes_{\mathcal{H}} K^{m} \otimes K^{m} \otimes K^{4+m} \\
= & \mathbf{1} \cdot K^{m} \otimes_{\mathcal{H}} 1 \otimes\left(K F \otimes E K^{3}-E K \otimes K^{3} F\right) \\
& -\left(q^{3}-q\right)^{-1} \mathbf{1} \otimes_{\mathcal{H}} 1 \otimes 1 \otimes K^{4}=F\left(\mathbf{1} \cdot K^{m}\right) .
\end{aligned}
$$


Let us next show that $F$ is a Hochschild 2-cocycle. To this end we note that $b\left(\mathbf{1} \otimes \mathcal{H} 1 \otimes E K \otimes K^{3} F\right)=\mathbf{1} \otimes_{\mathcal{H}} 1 \otimes 1 \otimes E K \otimes K^{3} F K^{2}$

$-\mathbf{1} \otimes \mathcal{H} 1 \otimes\left(1 \otimes E K+E K \otimes K^{2}\right) \otimes K^{3} F K^{2}$

$+\mathbf{1} \otimes \mathcal{H} 1 \otimes E K \otimes\left(K^{2} \otimes K^{3} F K^{2}+K^{3} F \otimes K^{2} K^{2}\right)$

$-\mathbf{1} \otimes \mathcal{H} 1 \otimes E K \otimes K^{3} F \otimes K^{2} K^{2}=0$,

and similarly that $b\left(\mathbf{1} \otimes \mathcal{H} 1 \otimes K F \otimes E K^{3}\right)=0$. As a result, $b(F)=0$. We next observe that

$$
\begin{aligned}
t\left(\mathbf{1} \otimes \mathcal{H} 1 \otimes E K \otimes K^{3} F\right) & =\mathbf{1} \otimes \mathcal{H} E K \otimes K^{3} F \otimes K^{2} K^{2} \\
& =\mathbf{1} \otimes_{\mathcal{H}} E \otimes K^{3} F K^{-1} \otimes K^{3} \\
& =-q^{-2} \mathbf{1} \otimes \mathcal{H} 1 \otimes E F K^{2} \otimes K^{4}-\mathbf{1} \otimes \mathcal{H} 1 \otimes K F \otimes E K^{3},
\end{aligned}
$$

where we used (5.1) in the second equality, and that

$$
\begin{aligned}
t\left(\mathbf{1} \otimes_{\mathcal{H}} 1 \otimes K F \otimes E K^{3}\right) & =\mathbf{1} \otimes \mathcal{H}_{\mathcal{H}} K F \otimes E K^{3} \otimes K^{4} \\
& =-q^{-2} \mathbf{1} \otimes_{\mathcal{H}} 1 \otimes F E K^{2} \otimes K^{4}-\mathbf{1} \otimes_{\mathcal{H}} 1 \otimes E K \otimes K^{3} F .
\end{aligned}
$$

As a result,

$$
\begin{aligned}
t\left(\mathbf{1} \otimes \mathcal{H} 1 \otimes\left(K F \otimes E K^{3}-E K \otimes K^{3} F\right)\right)= & \mathbf{1} \otimes \mathcal{H} 1 \otimes\left(K F \otimes E K^{3}-E K \otimes K^{3} F\right) \\
& +q^{-2} \mathbf{1} \otimes_{\mathcal{H}} 1 \otimes(E F-F E) K^{2} \otimes K^{4} \\
= & \mathbf{1} \otimes_{\mathcal{H}} 1 \otimes\left(K F \otimes E K^{3}-E K \otimes K^{3} F\right) \\
& +\left(q^{3}-q\right)^{-1} \mathbf{1} \otimes \mathcal{H} 1 \otimes K^{4} \otimes K^{4} \\
& -\left(q^{3}-q\right)^{-1} \mathbf{1} \otimes \mathcal{H} 1 \otimes 1 \otimes K^{4} .
\end{aligned}
$$

On the other hand, $b\left(\mathbf{1} \otimes_{\mathcal{H}} 1 \otimes 1 \otimes K^{4}\right)=0$, and $t\left(\mathbf{1} \otimes_{\mathcal{H}} 1 \otimes 1 \otimes K^{4}\right)=\mathbf{1} \otimes_{\mathcal{H}} 1 \otimes$ $K^{4} \otimes K^{4}$. Hence, we have

$$
b\left(\mathbf{1} \otimes \mathcal{H} 1 \otimes\left(K F \otimes E K^{3}-E K \otimes K^{3} F\right)-\left(q^{3}-q\right)^{-1} \mathbf{1} \otimes_{\mathcal{H}} 1 \otimes 1 \otimes K^{4}\right)=0
$$

and

$$
\begin{aligned}
& t\left(\mathbf{1} \otimes_{\mathcal{H}} 1 \otimes\left(K F \otimes E K^{3}-E K \otimes K^{3} F\right)-\left(q^{3}-q\right)^{-1} \mathbf{1} \otimes_{\mathcal{H}} 1 \otimes 1 \otimes K^{4}\right) \\
& \quad=\mathbf{1} \otimes_{\mathcal{H}} 1 \otimes\left(K F \otimes E K^{3}-E K \otimes K^{3} F\right)-\left(q^{3}-q\right)^{-1} \mathbf{1} \otimes_{\mathcal{H}} 1 \otimes 1 \otimes K^{4} .
\end{aligned}
$$

We thus conclude that

$F=1 \otimes_{\mathcal{H}} 1 \otimes\left(K F \otimes E K^{3}-E K \otimes K^{3} F\right)-\left(q^{3}-q\right)^{-1} \mathbf{1} \otimes \mathcal{H}^{1} \otimes 1 \otimes K^{4} \in C_{\mathcal{K}}^{2}(\mathcal{H}, V, N)$ is an equivariant cyclic 2-cocycle. 
Now, using the equivariant cup product (5.2) we obtain the following version of the Schmüdgen-Wagner 2-cocycle [34], see also [14].

Corollary 5.2 There is a nontrivial $\sigma^{-1}$-twisted cyclic 2-cocycle $\tau$ on $\mathcal{O}\left(S_{q}^{2}\right)$ such that

$$
\begin{aligned}
\tau\left(x_{0}, x_{1}, x_{2}\right)= & h\left(x_{0} K F\left(x_{1}\right) E K^{3}\left(x_{2}\right)\right)-h\left(x_{0} E K\left(x_{1}\right) K^{3} F\left(x_{2}\right)\right) \\
& -\left(q^{3}-q\right)^{-1} h\left(x_{0} x_{1} K^{4}\left(x_{2}\right)\right) .
\end{aligned}
$$

Proof We obtain the cocycle (5.4) by the cup product (5.2) of the Hopf-cyclic 0cocycle $[h] \in H C_{U_{q}\left(s u_{2}\right)}^{0}\left(\mathcal{O}\left(S_{q}^{2}\right),{ }^{-1} k\right)$ with the equivariant 2-cocycle (5.3).

We next show that it is nontrivial. Following [34], we consider the element

$$
\begin{aligned}
\eta^{\prime}= & q^{4} B^{*} \otimes A \otimes B+q^{2} B \otimes B^{*} \otimes A+q^{2} A \otimes B \otimes B^{*} \\
& -q^{2} B^{*} \otimes B \otimes A-q^{2} A \otimes B^{*} \otimes B-B \otimes A \otimes B^{*}+\left(q^{6}-q^{2}\right) A \otimes A \otimes A
\end{aligned}
$$

on which any Hochschild coboundary of a $\sigma^{-1}$-twisted cyclic 1-cocycle vanishes. Indeed, for any $\sigma^{-1}$-twisted cyclic 1-cocycle $\tau^{\prime} \in H C_{\sigma^{-1}}^{1}\left(\mathcal{O}\left(S_{q}^{2}\right)\right)$,

$$
b \tau^{\prime}\left(\eta^{\prime}\right)=\left(q^{4}-q^{2}\right) \tau^{\prime}(A \otimes A)=0 .
$$

On the other hand, a quick computation yields $\tau\left(\eta^{\prime}\right) \neq 0$.

Acknowledgements We would like to thank the anonymous referee whose careful reading and numerous suggestions greatly improved mathematical content and exposition of the article. We are grateful to the referee for alerting us about the existence of the unpublished note [27].

\section{References}

1. Brzeziński, T., Hajac, P.M.: Galois-type extensions and equivariant projectivity (2009). arXiv:0901.0141

2. Brzezinski, T., Wisbauer, R.: Corings and comodules. London Mathematical Society Lecture Note Series, vol. 309. Cambridge University Press, Cambridge (2003)

3. Connes, A.: Cohomologie cyclique et foncteurs $\mathrm{Ext}^{n}$. C. R. Acad. Sci. Paris Sér. I Math. 296(23), 953-958 (1983)

4. Connes, A.: Noncommutative differential geometry. Inst. Hautes Études Sci. Publ. Math. 62, 257-360 (1985)

5. Connes, A.: Noncommutative geometry. Academic Press Inc., San Diego (1994)

6. Connes, A., Moscovici, H.: Hopf algebras, cyclic cohomology and the transverse index theorem. Comm. Math. Phys. 198(1), 199-246 (1998)

7. Connes, A., Moscovici, H.: Background independent geometry and Hopf cyclic cohomology (2005). arXiv:math/0505475

8. Crainic, M.: Cyclic cohomology of Hopf algebras. J. Pure Appl. Algebra 166(1-2), 29-66 (2002)

9. Doi, Y.: Homological coalgebra. J. Math. Soc. Jpn. 33(1), 31-50 (1981)

10. Drupieski, C.M.: Cohomology rings for quantized enveloping algebras. Proc. Am. Math. Soc. 141(11), 3739-3753 (2013)

11. Farinati, M.A. Solotar, A.: Cyclic cohomology of coalgebras, coderivations and de Rham cohomology. In: Hopf algebras and quantum groups (Brussels, 1998), vol. 209 of Lecture Notes in Pure and Appl. Math., pp. 105-129. Dekker, New York (2000) 
12. Feng, P., Tsygan, B.: Hochschild and cyclic homology of quantum groups. Comm. Math. Phys. 140(3), 481-521 (1991)

13. Goodman, J., Krähmer, U.: Untwisting a twisted Calabi-Yau algebra. J. Algebra 406, 272-289 (2014)

14. Hadfield, T.: Twisted cyclic homology all Podleś quantum spheres. J. Geom. Phys. 57(2), 339-351 (2007)

15. Hajac, P.M., Khalkhali, M., Rangipour, B., Sommerhäuser, Y.: Hopf-cyclic homology and cohomology with coefficients. C. R. Math. Acad. Sci. Paris 338(9), 667-672 (2004)

16. Hajac, P.M., Khalkhali, M., Rangipour, B., Sommerhäuser, Y.: Stable anti-Yetter-Drinfeld modules. C. R. Math. Acad. Sci. Paris 338(8), 587-590 (2004)

17. Hochschild, G., Kostant, B., Rosenberg, A.: Differential forms on regular affine algebras. Trans. Am. Math. Soc. 102, 383-408 (1962)

18. Kaygun, A.: Products in Hopf-cyclic cohomology. Homol. Homotopy Appl. 10(2), 115-133 (2008)

19. Kaygun, A.: Uniqueness of pairings in Hopf-cyclic cohomology. J. K Theory 6(1), 1-21 (2010)

20. Kaygun, A.: Jacobi-Zariski exact sequence for Hochschild homology and cyclic (co)homology. Homol. Homotopy Appl. 14(1), 65-78 (2012)

21. Kaygun, A., Khalkhali, M.: Excision in Hopf cyclic homology. K Theory 37(1-2), 105-128 (2006)

22. Kaygun, A., Sütlü, S.: Hopf-cyclic cohomology of quantum enveloping algebras (2014). arXiv: 1409.4002

23. Khalkhali, M., Rangipour, B.: On the generalized cyclic Eilenberg-Zilber theorem. Can. Math. Bull. 47(1), 38-48 (2004)

24. Khalkhali, M., Rangipour, B.: Cup products in Hopf-cyclic cohomology. C. R. Math. Acad. Sci. Paris 340(1), 9-14 (2005)

25. Klimyk, A., Schmüdgen, K.: Quantum groups and their representations. Texts and Monographs in Physics. Springer, Berlin (1997)

26. Kustermans, J., Murphy, G.J., Tuset, L.: Differential calculi over quantum groups and twisted cyclic cocyles. J. Geom. Phys. 44(4), 570-594 (2003)

27. Kustermans, J., Rognes, J., Tuset, L.: The modular square for quantum groups. http://folk.uio.no/ rognes/papers/krt.ps

28. Kustermans, J., Rognes, J., Tuset, L.: The Connes-Moscovici approach to cyclic cohomology for compact quantum groups. K Theory 26(2), 101-137 (2002)

29. Loday, J.L.: Cyclic homology, vol. 301 of Grundlehren der Mathematischen Wissenschaften [Fundamental Principles of Mathematical Sciences], 2nd edn. Springer, Berlin (1998) (Appendix E by Maria O. Ronco, Chapter 13 by the author in collaboration with Teimuraz Pirashvili)

30. Masuda, T., Nakagami, Y., Watanabe, J.: Noncommutative differential geometry on the quantum SU(2). I. Algebraic Viewp. K Theory 4(2), 157-180 (1990)

31. Podleś, P.: Quantum spheres. Lett. Math. Phys. 14(3), 193-202 (1987)

32. Rangipour, B.: Cup products in Hopf cyclic cohomology via cyclic modules. Homol. Homotopy Appl. 10(2), 273-286 (2008)

33. Rangipour, B., Sütlü, S.: Characteristic classes of foliations via SAYD-twisted cocycles (2012). arXiv: 1210.5969

34. Schmüdgen, K., Wagner, E.: Dirac operator and a twisted cyclic cocyle on the standard Podleś quantum sphere. J. Reine Angew. Math. 574, 219-235 (2004)

35. Schneider, H.J.: Principal homogeneous spaces for arbitrary Hopf algebras. Isr. J. Math. 72(1-2), 167-195 (1990) (Hopf algebras) 